\title{
Noise Properties of Chord-Image Reconstruction
}

\author{
Dan Xia, Student Member, IEEE, Lifeng Yu, Emil Y. Sidky, Yu Zou, Nianming Zuo, and \\ Xiaochuan Pan*, Fellow, IEEE
}

\begin{abstract}
Recently, there has been much progress in algorithm development for image reconstruction in cone-beam computed tomography (CT). Current algorithms, including the chord-based algorithms, now accept minimal data sets for obtaining images on volume regions-of-interest (ROIs) thereby potentially allowing for reduction of X-ray dose in diagnostic CT. As these developments are relatively new, little effort has been directed at investigating the response of the resulting algorithm implementations to physical factors such as data noise. In this paper, we perform an investigation on the noise properties of ROI images reconstructed by using chord-based algorithms for different scanning configurations. We find that, for the cases under study, the chord-based algorithms yield images with comparable quality. Additionally, it is observed that, in many situations, large data sets contain extraneous data that may not reduce the ROI-image variances.
\end{abstract}

Index Terms-Chord, computed tomography (CT), cone-beam CT, noise, reconstruction.

\section{INTRODUCTION}

$\mathbf{I}$ $\mathrm{N}$ recent years, exact algorithms have been developed for reconstructing images [1] and for reconstructing images on " $\pi$-lines" [2]-[4] from helical cone-beam data. Since 2005, papers have being published on algorithm development for reconstructing images on chords for general trajectories [5]-[8]. Some of these algorithms can reconstruct images within 3-D regions of interest (ROIs) from cone-beam data containing both longitudinal and transverse truncations. The introduction of the $M$-line concept and reconstruction [5], [9] provides additional flexibility for covering volume ROIs.

As these algorithm developments are relatively recent, little effort has been directed at investigating their noise properties. With the algorithm development for ROI-image reconstruction, it has been tacitly assumed that the reduction in necessary scanning angle and in projection data may lead to ROI images from less radiation exposure. This conclusion may, however, depend

Manuscript received May 8, 2006; revised March 26, 2007. This work was supported by the National Institutes of Health under Grant EB00225 and Grant EB02765. The work of D. Xia was supported in part by Department of Defense (DoD) under Predoctoral Training Grant BC051553. The work of E. Y. Sidky was supported by the National Institutes of Health under Grant K01 EB003913. The work of N. Zuo was supported in part by the Natural Science Foundation of China under Grant 60328101. The contents are solely the responsibility of the authors and do not necessarily represent the official views of the National Institutes of Health. Asterisk indicates corresponding author.

D. Xia, L. Yu, E. Y. Sidky, and Y. Zou are with the Department of Radiology, The University of Chicago, Chicago, IL 60637 USA.

N. Zuo is with the National Laboratory of Pattern Recognition, Institute of Automation, Chinese Academy of Sciences, 100080 Beijing, China..

*X. Pan is with the Department of Radiology, The University of Chicago, Chicago, IL 60637 USA.

Color versions of some of the figures in this paper are available online at http://ieeexplore.ieee.org.

Digital Object Identifier 10.1109/TMI.2007.898567 on the noise properties of reconstruction algorithms. If ROI reconstruction from the minimal (or reduced) data set leads to noisier ROI images than reconstruction of the same ROI from a larger data set, it may be necessary to increase the X-ray source intensity for the ROI data set to attain the same image quality as those reconstructed from larger data sets. Such an increase can offset the fact that reduced or minimum projection data are needed for ROI reconstruction.

The focus of this paper is to investigate the noise properties of image reconstruction from minimal data set and large data set by use of chord-based algorithms. We demonstrate that the minimal data set can indeed lead to actual reduction of radiation exposure for attaining comparable image quality, defined in terms of image variance, as that obtained with a larger data set. In Section II, we briefly summarize the chord-based reconstruction algorithms: backprojection filtration (BPF) [2], [6], minimum data filtered backprojection (MDFBP) [4], [6], and filtered backprojection (FBP) [6], [10] algorithms. In Section III, we perform analysis and empirical studies on noise properties of images reconstructed from parallel-beam, fan-beam, and cone-beam data. Finally, a discussion is given in Section IV.

\section{CHORD-BASEd Reconstruction Algorithms}

We consider a continuous source trajectory specified by $\vec{r}_{0}(s)=(x(s), y(s), z(s))$, where $x(s), y(s)$, and $z(s)$ denote the $x-, y-$, and $z-$ components of $\vec{r}_{0}(s)$ in the fixed-coordinate system, and $s$ is a curve parameter indicating the position of the X-ray source on the trajectory. The projection data of the object function $f(\vec{r})$ can be mathematically expressed as

$$
D\left(\vec{r}_{0}(s), \hat{\beta}\right)=\int_{0}^{\infty} d t f\left(\vec{r}_{0}(s)+t \hat{\beta}\right)
$$

where the unit vector $\hat{\beta}$ denotes the direction of a specific X-ray passing through the point $\vec{r}$. We also introduce two additional coordinate systems $\{u, v, w\}$ and $\left\{u_{d}, v_{d}\right\}$ to describe the geometry in a general scan. They are fixed on the rotating source point and the cone-beam projection of the source point, respectively, which are referred to as the rotation-coordinate and detector-coordinate systems. Let $\hat{\mathrm{e}}_{u}(s), \hat{\mathrm{e}}_{v}(s)$, and $\hat{\mathrm{e}}_{w}(s)$ denote the orthogonal unit vectors of the rotation-coordinate system. The rotation-coordinate system can be chosen such that $\hat{\mathrm{e}}_{u}(s)$ and $\hat{\mathrm{e}}_{w}(s)$ are within the $x-y$ plane and $\hat{\mathrm{e}}_{v}(s)$ is parallel to the $z$ axis. One can also choose the "well oriented" coordinate system as the rotation-coordinate system [5], in which unit vector $\hat{\mathrm{e}}_{u}(s)$ is parallel to and unit vectors $\hat{\mathrm{e}}_{v}(s)$ and $\hat{\mathrm{e}}_{w}(s)$ 
are perpendicular to the direction of $\left(d \vec{r}_{0}(s) / d s\right)$. We assume that a detector plane is placed at a distance $S$ from the source point and orients along $\hat{\mathrm{e}}_{w}(s)$. The detector-coordinate system $\left\{u_{d}, v_{d}\right\}$ is the cone-beam projection of the 2-D coordinate system $\{u, v\}$ onto the detector plane, and the $u_{d}$ and $v_{d}$ axis are along $\hat{\mathrm{e}}_{u}(s)$ and $\hat{\mathrm{e}}_{v}(s)$, respectively. In this situation, we also use $P\left(u_{d}, v_{d}, s\right)$ to denote the cone-beam projection, thus $D\left(\vec{r}_{0}(s), \hat{\beta}\right)=P\left(u_{d}, v_{d}, s\right)$, when

$$
\begin{aligned}
\hat{\beta} & =\frac{1}{A\left(u_{d}, v_{d}\right)}\left[u_{d} \hat{\mathrm{e}}_{u}(s)+v_{d} \hat{\mathrm{e}}_{v}(s)-S \hat{\mathrm{e}}_{w}(s)\right] \\
\text { and } A\left(u_{d}, v_{d}\right) & =\sqrt{u_{d}^{2}+v_{d}^{2}+S^{2}}
\end{aligned}
$$

In a 2-D case, it can be observed that $v_{d}=0$. For notational convenience, we use $A\left(u_{d}\right)$ and $P\left(u_{d}, s\right)$ to denote $A\left(u_{d}, 0\right)$ and $P\left(u_{d}, 0, s\right)$, respectively.

A chord is a line segment connecting two points $\vec{r}_{0}\left(s_{a}\right)$ and $\vec{r}_{0}\left(s_{b}\right)$ on the trajectory. Any point $\vec{r}$ on the chord can be expressed as

$$
\vec{r}=\frac{\vec{r}_{0}\left(s_{a}\right)+\vec{r}_{0}\left(s_{b}\right)}{2}+x_{c} \hat{\mathrm{e}}_{c}, \quad x_{c} \in[-l, l]
$$

where $\hat{\mathrm{e}}_{c}=\left(\vec{r}_{0}\left(s_{b}\right)-\vec{r}_{0}\left(s_{a}\right)\right) /\left|\vec{r}_{0}\left(s_{b}\right)-\vec{r}_{0}\left(s_{a}\right)\right|$ denotes the direction of the chord, and $l=(1 / 2)\left|\vec{r}_{0}\left(s_{b}\right)-\vec{r}_{0}\left(s_{a}\right)\right|$ is one half of the chord length. For a helical trajectory, the curve parameter $s$ is linearly related to the rotation angle $\lambda$, and in the current work, we select $s=\lambda$. When $s_{a}$ and $s_{b}$ are within one turn, the chord becomes the conventional $\pi$-line segment [2], [11], [12]. The intersection between a chord and the object is referred to as a support segment. Let $x_{c 1}$ and $x_{c 2}$ represent the end points of a support segment. Because the trajectory under consideration never intersects the object, we have $\left[x_{c 1}, x_{c 2}\right] \subset[-l, l]$. Therefore, one can use $\left(x_{c}, s_{a}, s_{b}\right)$ and $f_{c}\left(x_{c}, s_{a}, s_{b}\right)$ to denote a point and the corresponding image on the chord. We have previously developed three algorithms, which are referred to as the BPF [2], [6], [10], MDFBP [4], [6], and FBP [6], [10] algorithms, respectively, for exact image reconstruction on a chord of a general trajectory.

\section{A. BPF Algorithm}

The BPF algorithm [2], [6] reconstructs the image on a chord specified by $s_{a}$ and $s_{b}$ as

$$
\begin{aligned}
f_{c}\left(x_{c}, s_{a}, s_{b}\right)=\hat{f}\left(x_{c}, s_{a}, s_{b}\right)+\frac{1}{2 \pi} \frac{P\left(u_{d 0}, v_{d 0}, s_{a}\right)}{b\left(x_{c}\right)} \\
\times\left[\frac{\sqrt{\left(l-x_{B}\right)\left(l-x_{A}\right)}}{l-x_{c}}+\frac{\sqrt{\left(l+x_{A}\right)\left(l+x_{B}\right)}}{l+x_{c}}\right]
\end{aligned}
$$

where $x_{c} \in\left[x_{A}, x_{B}\right]$, and parameters $x_{A}$ and $x_{B}$ are two points on the chord satisfying $\left[x_{c 1}, x_{c 2}\right] \subseteq\left[x_{A}, x_{B}\right] \subset[-l, l]$. The function $b\left(x_{c}\right)$ is defined as $b\left(x_{c}\right)=\sqrt{\left(x_{B}-x_{c}\right)\left(x_{c}-x_{A}\right)}$, and $P\left(u_{d 0}, v_{d 0}, s_{a}\right)$ denotes the projection along the chord specified by $s_{a}$ and $s_{b}$. The filtered image $\hat{f}\left(x_{c}, s_{a}, s_{b}\right)$ is given by

$$
\hat{f}\left(x_{c}, s_{a}, s_{b}\right)=\frac{1}{2 \pi^{2}} \frac{1}{b\left(x_{c}\right)} \int_{\mathbf{R}} \frac{d x_{c}^{\prime}}{x_{c}-x_{c}^{\prime}} b\left(x_{c}^{\prime}\right) g\left(x_{c}^{\prime}, s_{a}, s_{b}\right)
$$

where the backprojection image on the chord can be written as

$$
\begin{aligned}
g\left(x_{c}^{\prime}, s_{a}, s_{b}\right)= & \Pi_{c}\left(x_{c}^{\prime}\right) \int_{s_{a}}^{s_{b}} d s \frac{\operatorname{sgn}\left(-\hat{\beta} \cdot \hat{\mathrm{e}}_{w}\right)}{\left|\vec{r}^{\prime}-\vec{r}_{0}(s)\right|^{2}} \\
\times & \left\{-\frac{d \vec{r}_{0}(s)}{d s} \cdot \hat{\beta} P\left(u_{d}, v_{d}, s\right)\right. \\
& +\left[\frac{d \vec{r}_{0}(s)}{d s} \cdot \hat{\mathrm{e}}_{u}(s)+\frac{u_{d}}{S(s)} \frac{d \vec{r}_{0}(s)}{d s} \cdot \hat{\mathrm{e}}_{w}(s)\right] \\
& \times A\left(u_{d}, v_{d}\right) \frac{\partial P\left(u_{d}, v_{d}, s\right)}{\partial u_{d}} \\
& +\left[\frac{d \vec{r}_{0}(s)}{d s} \cdot \hat{\mathrm{e}}_{v}(s)+\frac{v_{d}}{S(s)} \frac{d \vec{r}_{0}(s)}{d s} \cdot \hat{\mathrm{e}}_{w}(s)\right] \\
& \left.\times A\left(u_{d}, v_{d}\right) \frac{\partial P\left(u_{d}, v_{d}, s\right)}{\partial v_{d}}\right\}
\end{aligned}
$$

and the rect function $\Pi_{c}\left(x_{c}^{\prime}\right)=1$ if $x_{c}^{\prime} \in\left[x_{A}, x_{B}\right]$ and zero otherwise. It can be observed in (4) that the chord image can be obtained exactly from knowledge of the backprojection image $g\left(x_{c}^{\prime}, s_{a}, s_{b}\right)$ for $x_{c}^{\prime} \in\left[x_{A}, x_{B}\right]$, which we refer to as the reconstruction segment because it determines the actual reconstruction interval on the chord. In particular, because the reconstruction segment $\left[x_{A}, x_{B}\right]$ can be chosen as small as the support segment $\left[x_{c 1}, x_{c 2}\right]$, the chord image can be reconstructed from knowledge of $g\left(x_{c}^{\prime}, s_{a}, s_{b}\right)$ only on the support segment. This interesting property of the Hilbert transform forms the basis for exact image reconstruction on a chord from projections containing longitudinal or transverse truncations [13].

\section{B. MDFBP Algorithm}

The BPF algorithm reconstructs the chord image by performing a 1-D filtration [i.e., the integration over $x_{c}^{\prime}$ in (4)] of the backprojection image [i.e., the integration over $s$ in (6)]. On the other hand, the MDFBP algorithm [4], [6] reconstructs the chord image by performing a 1-D data filtration (i.e., the integration over $u_{c}^{\prime}$ ) prior to their backprojection (i.e., the integration over $s$ ) onto the chord

$$
\begin{aligned}
f_{c}\left(x_{c}, s_{a}, s_{b}\right) & \\
= & \frac{1}{2 \pi^{2}} \frac{1}{b\left(x_{c}\right)} \int_{s_{a}}^{s_{b}} d s\left[w_{2}\left(1-u_{c}\right)+w_{1} u_{c}\right] \int_{\mathbb{R}} \frac{d u_{c}^{\prime}}{u_{c}-u_{c}^{\prime}} P_{\Pi} \\
& +\frac{1}{2 \pi} \frac{P\left(u_{d 0}, v_{d 0}, s_{a}\right)}{b\left(x_{c}\right)} \\
& \times\left[\frac{\sqrt{\left(l-x_{B}\right)\left(l-x_{A}\right)}}{l-x_{c}}+\frac{\sqrt{\left(l+x_{A}\right)\left(l+x_{B}\right)}}{l+x_{c}}\right]
\end{aligned}
$$


where the modified data function is given by

$$
\begin{aligned}
P_{\Pi}= & \Pi_{c}\left(x_{c}^{\prime}\right) \frac{b\left(x_{c}^{\prime}\right)}{w_{2}\left(1-u_{c}^{\prime}\right)+w_{1} u_{c}^{\prime}} \frac{\operatorname{sgn}\left(-\hat{\beta} \cdot \hat{\mathrm{e}}_{w}\right)}{\left|\vec{r}^{\prime}-\vec{r}_{0}(s)\right|^{2}} \\
\times & -\frac{d \vec{r}_{0}(s)}{d s} \cdot \hat{\beta} P\left(u_{d}, v_{d}, s\right) \\
& +\left[\frac{d \vec{r}_{0}(s)}{d s} \cdot \hat{\mathrm{e}}_{u}(s)+\frac{u_{d}}{S(s)} \frac{d \vec{r}_{0}(s)}{d s} \cdot \hat{\mathrm{e}}_{w}(s)\right] \\
& \times A\left(u_{d}, v_{d}\right) \frac{\partial P\left(u_{d}, v_{d}, s\right)}{\partial u_{d}} \\
& +\left[\frac{d \vec{r}_{0}(s)}{d s} \cdot \hat{\mathrm{e}}_{v}(s)+\frac{v_{d}}{S(s)} \frac{d \vec{r}_{0}(s)}{d s} \cdot \hat{\mathrm{e}}_{w}(s)\right] \\
& \left.\times A\left(u_{d}, v_{d}\right) \frac{\partial P\left(u_{d}, v_{d}, s\right)}{\partial v_{d}}\right\}
\end{aligned}
$$

$w_{1}=-\left[\vec{r}_{0}\left(s_{a}\right)-\vec{r}_{0}(s)\right] \cdot \hat{\mathrm{e}}_{w}$, and $w_{2}=-\left[\vec{r}_{0}\left(s_{b}\right)-\vec{r}_{0}(s)\right] \cdot$ $\hat{\mathrm{e}}_{w}$. For a source position $s$, the variables $u_{c}$ and $u_{c}^{\prime}$ denote the cone-beam projections of $x_{c}$ and $x_{c}^{\prime}$ onto the detector and can be obtained, respectively, by replacing $x$ with $x_{c}$ and $x_{c}^{\prime}$ in

$$
u=\frac{w_{2}(x+l)}{w_{1}(l-x)+w_{2}(x+l)} .
$$

The rect function $\Pi_{c}\left(x_{c}^{\prime}\right)$ in (8) indicates that the MDFBP algorithm can reconstruct a chord image from knowledge of data only on the cone-beam projection of the reconstruction segment $\left[x_{A}, x_{B}\right]$, which can be as small as the support segment. Therefore, similar to the BPF algorithm, the MDFBP algorithm can also reconstruct a chord image from data containing truncations [4], [6].

\section{FBP Algorithm}

The chord-based FBP algorithm [6], [10] can be expressed as

$$
\begin{aligned}
& f_{c}\left(x_{c}, s_{a}, s_{b}\right)=\frac{1}{2 \pi^{2}} \int_{s_{a}}^{s_{b}} d s \frac{A}{\left|\vec{r}-\vec{r}_{0}(s)\right|} \\
& \times\left.\int_{-\infty}^{\infty} \frac{d u_{c}^{\prime}}{u_{c}-u_{c}^{\prime}} \frac{1}{\left|\vec{r}^{\prime}-\vec{r}_{0}(s)\right|} \frac{\partial}{\partial q} D\left(\vec{r}_{0}(q), \hat{\beta}\right)\right|_{q=s}
\end{aligned}
$$

where $u_{c}$ indicates the cone-beam projection of $x_{c}$ onto the detector and is determined by using $x_{c}$ to replace $x$ in (8), and $A$ denotes the distance from the source point $\vec{r}_{0}(s)$ to a point on the detector at which the ray connecting $\vec{r}$ and $\vec{r}_{0}(s)$ intersects the detector. As the filtering (i.e., the integration over $u_{c}^{\prime}$ ) is carried out over the projection of the straight line containing the chord, similar to other existing FBP-based algorithms, the chord-based FBP algorithm cannot exactly reconstruct ROI images from data containing transverse truncations.

\section{Data-Sufficiency Conditions}

As shown in (9), a data-sufficiency condition for the FBP algorithm is: 1) data are available over the trajectory segment $s \in\left[s_{a}, s_{b}\right]$ and 2) for each $s$, data on the cone-beam projection of the chord are nontruncated. This condition is similar to that for other FBP-based algorithms [1], [9], [14], [15], [16]. From (4) and (7), a data-sufficiency condition for the chord-based
BPF and MDFBP algorithms is: 1) data are collected over the trajectory segment $\left[s_{a}, s_{b}\right]$ and 2 ) at each $s$, data only on the cone-beam projection of the reconstruction segment $\left[x_{A}, x_{B}\right]$ on the chord are available. It follows that, because the reconstruction segment $\left[x_{A}, x_{B}\right]$ can be chosen as small as the support segment $\left[x_{c 1}, x_{c 2}\right]$, the BPF and MDFBP algorithms require, at each $s$, data only on the cone-beam projection of the support segment $\left[x_{c 1}, x_{c 2}\right]$ (instead of the entire chord-line as the chord-based FBP algorithm requires). Different selections of the reconstruction segment $\left[x_{A}, x_{B}\right]$ imply that different amounts of data at each $s$ can be used for reconstructing the chord image. Under the ideal continuous conditions, different selections of $\left[x_{A}, x_{B}\right]$ yield identical chord images. However, when data contain noise and other inconsistencies, and when different selections of $\left[x_{A}, x_{B}\right]$ are used, the BPF and MDFBP algorithms in their discrete forms may yield different chord images. This is an issue that will be investigated in the following.

\section{NOISE PROPERTIES OF CHORD-BASED IMAGE RECONSTRUCTION}

The BPF, MDFBP, and FBP algorithms described above can be applied to reconstructing chord images from parallel-, fan-, and cone-beam data [17]. Algorithms analogous to the BPF algorithm that are capable of reconstructing 2-D ROI images from truncation data have also previously been proposed [8], [13], [18]. We study the noise properties of chord-based reconstruction by use of these algorithms in their discrete forms. As mentioned above, the BPF and MDFBP algorithms can reconstruct the image on the reconstruction segment $\left[x_{A}, x_{B}\right]$ as long as it covers the support segment $\left[x_{c 1}, x_{c 2}\right]$. We analyze image-noise properties on reconstruction segments of different lengths.

\section{A. Analysis of Image-Noise Properties}

The chord-based algorithms invoke three major mathematical operations: differentiation, backprojection, and filtration. To a large extent, the BPF, MDFBP, and FBP algorithms differ in the orders of invoking these operations. Below, we focus on investigating the noise properties of differentiation, backprojection, and filtration in the BPF algorithm. The approach taken in the investigation is readily applicable to analyzing the noise properties of the MDFBP and FBP algorithms. In the presence of data noise, the measured projection $\mathbf{D}\left(\vec{r}_{0}(s), \hat{\beta}\right)$ should be interpreted as a stochastic process. (Throughout the paper, we use boldface and normal letters to denote a stochastic process and its mean, respectively.) Because the backprojection $\mathrm{g}\left(x_{c}, s_{a}, s_{b}\right)$ and the final image $\mathbf{f}_{c}\left(x_{c}, s_{a}, s_{b}\right)$ on a chord are computed from $\mathbf{D}\left(\vec{r}_{0}(s), \hat{\beta}\right)$, they should also be considered as stochastic processes. We focus in this section on investigating the chord-image variance $\mathbf{f}_{c}\left(x_{c}, s_{a}, s_{b}\right)$ by the investigation of noise propagation through each step involved in the BPF reconstruction algorithm.

1) Noise Properties of Differentiation/Backprojection for Parallel-Beam Data: Let $\mathbf{P}\left(u_{d}, s\right)$ denote the parallel-beam projection on detector bin $u_{d}$ acquired at view $s$. We assume the covariance of the projection data $\mathbf{P}\left(u_{d}, s\right)$ to be uncorrelated, i.e.,

$$
\operatorname{Cov}\left\{\mathbf{P}\left(u_{d}, s\right), \mathbf{P}\left(u_{d}^{\prime}, s^{\prime}\right)\right\}=\sigma^{2}\left(u_{d}, s\right) \delta\left(u_{d}-u_{d}^{\prime}\right) \delta\left(s-s^{\prime}\right)
$$


where $\sigma^{2}\left(u_{d}, s\right)$ denotes the variance of the projection data. The backprojection image on the chord is given by [13]

$$
\mathbf{g}\left(x_{c}, s_{a}, s_{b}\right)=\int_{-\frac{\pi}{2}}^{\frac{\pi}{2}} d s \frac{\partial}{\partial u_{d}} \mathbf{P}\left(u_{d}, s\right)
$$

where $u_{d}=\vec{r} \cdot \hat{\mathrm{e}}_{u}(s)$. The final image variances on a chord depend upon the covariance of the backprojection image, which, using (11), can be written as

$$
\begin{aligned}
& \operatorname{Cov}\left\{\mathbf{g}\left(x_{c}, s_{a}, s_{b}\right), \mathbf{g}\left(x_{c}^{\prime}, s_{a}, s_{b}\right)\right\} \\
& =\int_{-\frac{\pi}{2}}^{\frac{\pi}{2}} d s \int_{-\frac{\pi}{2}}^{\frac{\pi}{2}} d s^{\prime} \operatorname{Cov}\left\{\frac{\partial}{\partial u_{d}} \mathbf{P}\left(u_{d}, s\right), \frac{\partial}{\partial u_{d}^{\prime}} \mathbf{P}\left(u_{d}^{\prime}, s^{\prime}\right)\right\} .
\end{aligned}
$$

The evaluation of the backprojection-image covariance involves the data-derivative covariance, which can be conveniently written as

$$
\begin{aligned}
\operatorname{Cov}\left\{\frac{\partial}{\partial u_{d}} \mathbf{P}\left(u_{d}, s\right), \frac{\partial}{\partial u_{d}^{\prime}} \mathbf{P}\left(u_{d}^{\prime}, s^{\prime}\right)\right\}=a \omega \sigma^{2}\left(u_{d}, s\right) \\
\times \delta\left(u_{d}-u_{d}^{\prime}\right) \delta\left(s-s^{\prime}\right)+\mathrm{T}_{\text {para }}\left(u_{d}, u_{d}^{\prime}, s, s^{\prime}\right)
\end{aligned}
$$

where $\operatorname{Var}\left\{\mathbf{P}\left(u_{d}, s\right)\right\}=\sigma^{2}\left(u_{d}, s\right)$ denotes the known data variance, which can be a function of $u_{d}$ and $s$. The second term $\mathrm{T}_{\text {para }}\left(u_{d}, u_{d}^{\prime}, s, s^{\prime}\right)$ represents the difference between the term on the left-hand side and the first term on the right-hand side of (13). Although the magnitude of $\mathrm{T}_{\text {para }}\left(u_{d}, u_{d}^{\prime}, s, s^{\prime}\right)$ can be larger than or comparable to that of the first term in the right-hand side of (13), numerical results that follow show that its contribution to the final image variance on a chord is negligibly small. Therefore, we consider only the first term in the derivation of the chord-image variance below. The parameters $a$ and $\omega$ are introduced to account for the interpolation effect of the discrete data derivative and discrete backprojection on the chord-image variance. Using the first term in (13), we can rewrite (12) as

$$
\begin{aligned}
\operatorname{Cov}\left\{\mathbf{g}\left(x_{c}, s_{a}, s_{b}\right), \mathbf{g}\right. & \left.\left(x_{c}^{\prime}, s_{a}, s_{b}\right)\right\} \\
& \approx a \omega \int_{-\frac{\pi}{2}}^{\frac{\pi}{2}} d s \sigma^{2}\left(u_{d}, s\right) \delta\left(u_{d}-u_{d}^{\prime}\right) .
\end{aligned}
$$

We now consider two points $x_{c}$ and $x_{c}^{\prime}$ on the chord and let $u_{d}$ and $u_{d}^{\prime}$ denote their parallel-beam or fan-beam projections onto the detector. Clearly, for a source position $s$ that satisfies $s \neq s_{a}$ or $s_{b}$, one can conclude that

$$
\begin{array}{lll}
u_{d}-u_{d}^{\prime}=0 & \text { if } & x_{c}-x_{c}^{\prime}=0 \\
u_{d}-u_{d}^{\prime} \neq 0 & \text { if } & x_{c}-x_{c}^{\prime} \neq 0 .
\end{array}
$$

Thus, if $x_{c}=x_{c}^{\prime}, \operatorname{Cov}\left\{\mathrm{g}\left(x_{c}, s_{a}, s_{b}\right), \mathbf{g}\left(x_{c}, s_{a}, s_{b}\right)\right\}=$ $\delta(0) a \omega \int_{-\pi / 2}^{\pi / 2} d s \sigma^{2}\left(u_{d}, s\right)$ and, if $x_{c} \neq x_{c}^{\prime}$,
$\operatorname{Cov}\left\{\mathbf{g}\left(x_{c}, s_{a}, s_{b}\right), \mathbf{g}\left(x_{c}^{\prime}, s_{a}, s_{b}\right)\right\}=0$. Therefore, the covariance of the backprojection image for parallel-beam projections can be re-expressed as

$$
\operatorname{Cov}\left\{\mathrm{g}\left(x_{c}, s_{a}, s_{b}\right), \mathrm{g}\left(x_{c}^{\prime}, s_{a}, s_{b}\right)\right\} \approx c\left(x_{c}\right) \delta\left(x_{c}-x_{c}^{\prime}\right)
$$

where

$$
c\left(x_{c}\right)=a \omega \int_{-\frac{\pi}{2}}^{\frac{\pi}{2}} d s \sigma^{2}\left(u_{d}, s\right) .
$$

2) Noise Properties of Differentiation/Backprojection for Fan-Beam Data: In the fan-beam case, we use $\mathbf{P}\left(u_{d}, s\right)$ to denote the projection on detector bin $u_{d}$ acquired at view $s$. Again, we assume $\mathbf{P}\left(u_{d}, s\right)$ to be uncorrelated and to satisfy (10). The backprojection image in (6) can be re-expressed as

$$
\mathrm{g}\left(x_{c}, s_{a}, s_{b}\right)=\int_{s_{a}}^{s_{b}} d s \frac{1}{\left|\vec{r}-\vec{r}_{0}(s)\right|^{2}} \mathbf{P}^{\prime}\left(u_{d}, s\right)
$$

where $u_{d}=\left(S \vec{r} \cdot \hat{\mathrm{e}}_{u}(s) /\left(\vec{r}_{0}(s)-\vec{r}\right) \cdot \hat{\mathrm{e}}_{w}(s)\right)$ is the fan-beam projection of $x_{c}$ onto the detector. The weighted-data derivative $\mathbf{P}^{\prime}\left(u_{d}, s\right)$ is given by

$$
\mathbf{P}^{\prime}\left(u_{d}, s\right)=A^{2}\left(u_{d}\right)\left|\frac{d \vec{r}_{0}(s)}{d s}\right| \frac{\partial}{\partial u_{d}}\left[\frac{\mathbf{P}\left(u_{d}, s\right)}{A\left(u_{d}\right)}\right] .
$$

Using (18), one can write the covariance of the backprojection image as

$$
\begin{aligned}
\operatorname{Cov} & \left\{\mathbf{g}\left(x_{c}, s_{a}, s_{b}\right), \mathbf{g}\left(x_{c}^{\prime}, s_{a}, s_{b}\right)\right\} \\
= & \int_{s_{a}}^{s_{b}} d s \int_{s_{a}}^{s_{b}} d s^{\prime} \frac{1}{\left|\vec{r}-\vec{r}_{0}(s)\right|^{2}} \frac{1}{\left|\vec{r}-\vec{r}_{0}\left(s^{\prime}\right)\right|^{2}} \\
& \times \operatorname{Cov}\left\{\mathbf{P}^{\prime}\left(u_{d}, s\right), \mathbf{P}^{\prime}\left(u_{d}^{\prime}, s^{\prime}\right)\right\}
\end{aligned}
$$

which depends upon the covariance of $\mathbf{P}^{\prime}\left(u_{d}, s\right)$. Again, we can conveniently write the covariance of $\mathbf{P}^{\prime}\left(u_{d}, s\right)$ as

$$
\begin{aligned}
\operatorname{Cov} & \left\{\mathbf{P}^{\prime}\left(u_{d}, s\right), \mathbf{P}^{\prime}\left(u_{d}^{\prime}, s^{\prime}\right)\right\} \\
= & a \omega \sigma^{2}\left(u_{d}, s\right) A^{2}\left(u_{d}\right)\left|\frac{d \vec{r}_{0}(s)}{d s}\right|^{2} \delta\left(u_{d}-u_{d}^{\prime}\right) \delta\left(s-s^{\prime}\right) \\
& +\mathrm{T}_{f a n}\left(u_{d}, u_{d}^{\prime}, s, s^{\prime}\right)
\end{aligned}
$$

where $\operatorname{Var}\left\{\mathbf{P}\left(u_{d}, s\right)\right\}=\sigma^{2}\left(u_{d}, s\right)$ denotes the known data variance, which can be a function of $u_{d}$ and $s$. The second term $\mathrm{T}_{f a n}\left(u_{d}, u_{d}^{\prime}, s, s^{\prime}\right)$ represents the difference between the term on the left-hand side and the first term in the right-hand side of (21). As numerical results indicate, it turns out that $\mathrm{T}_{f a n}\left(u_{d}, u_{d}^{\prime}, s, s^{\prime}\right)$ will also have a negligible contribution to the chord-image variance. Therefore, we consider only the first term in the derivation of the chord-image variance below. Again, the parameters $a$ and $\omega$ are introduced to account for the interpolation effect of the discrete data derivative and discrete 
backprojection on the chord-image variance. Using the first term in (21), we can rewrite (20) as

$$
\begin{aligned}
& \operatorname{Cov}\left\{\mathbf{g}\left(x_{c}, s_{a}, s_{b}\right), \mathbf{g}\left(x_{c}^{\prime}, s_{a}, s_{b}\right)\right\} \\
& \approx a \omega \int_{s_{a}}^{s_{b}} d s \frac{\sigma^{2}\left(u_{d}, s\right) A^{2}\left(u_{d}\right)}{\left|\vec{r}-\vec{r}_{0}(s)\right|^{4}}\left|\frac{d \vec{r}_{0}(s)}{d s}\right|^{2} \delta\left(u_{d}-u_{d}^{\prime}\right) .
\end{aligned}
$$

Similar to the parallel-beam case described previously, using (15), one can conclude that

$$
\operatorname{Cov}\left\{\mathbf{g}\left(x_{c}, s_{a}, s_{b}\right), \mathbf{g}\left(x_{c}^{\prime}, s_{a}, s_{b}\right)\right\} \approx c\left(x_{c}\right) \delta\left(x_{c}-x_{c}^{\prime}\right)
$$

where

$$
c\left(x_{c}\right)=a \omega \int_{s_{a}}^{s_{b}} d s \frac{A^{2}\left(u_{d}\right)}{\left|\vec{r}-\vec{r}_{0}(s)\right|^{4}}\left|\frac{d \vec{r}_{0}(s)}{d s}\right|^{2} \sigma^{2}\left(u_{d}, s\right) .
$$

3) Noise Properties of Differentiation/Backprojection for Cone-Beam Data: In the cone-beam case, let $\mathbf{P}\left(u_{d}, v_{d}, s\right)$ denote the projection at view $s$ on a detector bin specified by $\left(u_{d}, v_{d}\right)$. In the so-called "well oriented" rotation-coordinate system [5], unit vector $\hat{\mathrm{e}}_{u}(s)$ is parallel to and unit vectors $\hat{\mathrm{e}}_{v}(s)$ and $\hat{\mathrm{e}}_{w}(s)$ are orthogonal to the tangential direction $\left(d \vec{r}_{0}(s) / d s\right)$ of the source trajectory. Let $u_{d}$ and $v_{d}$ denote the coordinates along $\hat{\mathrm{e}}_{u}(s)$ and $\hat{\mathrm{e}}_{v}(s)$. It can be shown [5] that the backprojection image depends only upon the data derivative along $u_{d}$. Therefore, the reconstruction formula for the cone-beam backprojection image can be obtained from that for the fan-beam backprojection image in (18) by simply replacing $P\left(u_{d}, s\right)$ and $A\left(u_{d}\right)$ with $P\left(u_{d}, v_{d}, s\right)$ and $A\left(u_{d}, v_{d}\right)$, respectively. Subsequently, one can show that the covariance of the cone-beam backprojection image $\mathrm{g}\left(x_{c}, s_{a}, s_{b}\right)$ also satisfies (23) and (24).

4) Estimation of Parameters $a$ and $\omega$ in Discrete Form: The parameter $a$ is introduced to account for the interpolation effect of the discrete data-derivative on the chord-image variance. We consider a two-point derivative, which was used in our numerical studies. Let $\mathbf{P}_{i}$ denote the discrete data, where $i=$ $1,2, \ldots, I$, and $I$ indicates the total number of data points. We assume that data $\mathbf{P}_{i}$ are uncorrelated and use $\operatorname{Var}\left\{\mathbf{P}_{i}\right\}$ to denote their variances. The two-point data derivative is defined as

$$
\overline{\mathbf{P}}_{i}=\frac{1}{2}\left[\mathbf{P}_{i+1}-\mathbf{P}_{i-1}\right]
$$

Therefore, the variance of the discrete data derivative $\overline{\mathbf{P}}_{i}$ can be written as

$$
\operatorname{Var}\left\{\overline{\mathbf{P}}_{i}\right\}=\frac{1}{2} \frac{\operatorname{Var}\left\{\mathbf{P}_{i+1}\right\}+\operatorname{Var}\left\{\mathbf{P}_{i-1}\right\}}{2} .
$$

When data variances are identical, (26) becomes

$$
\operatorname{Var}\left\{\overline{\mathbf{P}}_{i}\right\}=\frac{1}{2} \operatorname{Var}\left\{\mathbf{P}_{i}\right\}
$$

Therefore, in our studies, we select $a=1 / 2$, which is the coefficient in (27).

The parameter $\omega$ was introduced to account for the interpolation effect of discrete backprojection on the chord-image variances. The estimated value of $\omega$ depends obviously upon the specific interpolation scheme used in the discrete backprojection. We illustrate our estimation of $\omega$ when a two-point interpolation is used for the discrete backprojection in the paralleland fan-beam cases. One can readily obtain estimates of $\omega$ when other interpolation schemes are used. In our studies, $u_{d}$ is measured in the unit of detector-bin size. Let $i=f$ foor $\left(u_{d}\right)+I_{0}$, where $I_{0}$ denotes the index corresponding to $u_{d}=0$. At a backprojection view $s$, we use $\mathbf{P}_{i}^{\prime}$ to denote the discrete weigheddata derivatives. For a given $u_{d}$, we express the interpolated weighted-data derivative as

$$
\mathbf{P}_{u_{d}}^{\prime}=(1-\gamma) \mathbf{P}_{i}^{\prime}+\gamma \mathbf{P}_{i+1}^{\prime}
$$

where $\gamma=u_{d}-i$. Furthermore, we can write the variance of $\mathbf{P}_{u_{d}}^{\prime}$ as

$$
\operatorname{Var}\left\{\mathbf{P}_{u_{d}}^{\prime}\right\} \approx\left[(1-\gamma)^{2}+\gamma^{2}\right] \operatorname{Var}\left\{\mathbf{P}_{i}^{\prime}\right\}
$$

For the sake of simplifying the estimation of $\omega$, we have ignored the correlation between $\mathbf{P}_{i}^{\prime}$ and $\mathbf{P}_{i+1}^{\prime}$ and assumed that $\operatorname{Var}\left\{\mathbf{P}_{i}^{\prime}\right\} \approx \operatorname{Var}\left\{\mathbf{P}_{i+1}^{\prime}\right\}$. We select $\omega$ as the average over all of the possible $\gamma \mathrm{s}$, which can be computed as

$$
\omega=\int_{0}^{1}\left[(1-\gamma)^{2}+\gamma^{2}\right] d \gamma=\frac{2}{3}
$$

Finally, with a substitution of $a=1 / 2$ and $\omega=2 / 3$ into (17) and (24), we obtain the variances $c\left(x_{c}\right)$ of the backprojection images on the chords for the parallel-beam and fan-beam projections, respectively, as

$$
\begin{aligned}
& c\left(x_{c}\right)=\frac{1}{3} \int_{-\frac{\pi}{2}}^{\frac{\pi}{2}} d s \sigma^{2}\left(u_{d}, s\right) \\
& c\left(x_{c}\right)=\frac{1}{3} \int_{s_{a}}^{s_{b}} d s \frac{A^{2}\left(u_{d}\right)}{\left|\vec{r}-\vec{r}_{0}(s)\right|^{4}}\left|\frac{d \vec{r}_{0}(s)}{d s}\right|^{2} \sigma^{2}\left(u_{d}, s\right) .
\end{aligned}
$$

5) Noise Property of Weighted Hilbert Transform Over a Finite Interval: The weighted Hilbert transform constitutes an important step in the chord-based BPF, MDFBP, and FBP algorithms. Consequently, the noise properties of these algorithms depend upon that of the weighted Hilbert transform, which we study in the following. Let $\hat{f}\left(x_{c}, s_{a}, s_{b}\right)$ denote the weighted Hilbert transform of the backprojection image $g\left(x_{c}, s_{a}, s_{b}\right)$

$$
\hat{f}\left(x_{c}, s_{a}, s_{b}\right)=\frac{1}{b\left(x_{c}\right)} \int_{x_{A}}^{x_{B}} \frac{d x_{c}^{\prime}}{x_{c}-x_{c}^{\prime}} b\left(x_{c}^{\prime}\right) g\left(x_{c}^{\prime}, s_{a}, s_{b}\right) .
$$

We assume that $g\left(x_{c}^{\prime}, s_{a}, s_{b}\right)$ is band-limited to $\nu_{m}$. Therefore, the Hilbert transform kernel $\left(1 / x_{c}\right)$ can be replaced by

$$
h\left(x_{c}\right)=-\pi j \int_{-\nu_{m}}^{\nu_{m}} d \nu \operatorname{sgn}[\nu] \mathrm{e}^{2 \pi j \nu x_{c}}=\frac{2 \sin ^{2}\left(\pi \nu_{m} x_{c}\right)}{x_{c}} .
$$

In the presence of noise, the weighted Hilbert transform $\hat{\mathbf{f}}\left(x_{c}, s_{a}, s_{b}\right)$ should be interpreted as a stochastic process, 
which is denoted in boldface. The variance of $\hat{\mathbf{f}}\left(x_{c}, s_{a}, s_{b}\right)$ can be written as

$$
\begin{aligned}
\operatorname{Var}\left\{\hat{\mathbf{f}}\left(x_{c}, s_{a}, s_{b}\right)\right\}= & \frac{1}{b^{2}\left(x_{c}\right)} \int_{x_{A}}^{x_{B}} d x_{c}^{\prime} \int_{x_{A}}^{x_{B}} d x_{c}^{\prime \prime} b\left(x_{c}^{\prime}\right) b\left(x_{c}^{\prime \prime}\right) \\
& \times h\left(x_{c}-x_{c}^{\prime}\right) h\left(x_{c}-x_{c}^{\prime \prime}\right) \\
& \times \operatorname{Cov}\left\{\mathbf{g}\left(x_{c}^{\prime}, s_{a}, s_{b}\right), \mathbf{g}\left(x_{c}^{\prime \prime}, s_{a}, s_{b}\right)\right\} .
\end{aligned}
$$

As (16) and (23) show, the backprojection image $\mathrm{g}\left(x_{c}, s_{a}, s_{b}\right)$ can be treated as an approximated uncorrelated stochastic process. Using the result of (16) or (23), we can write (35) as

$$
\begin{aligned}
& \operatorname{Var}\left\{\hat{\mathbf{f}}\left(x_{c}, s_{a}, s_{b}\right)\right\} \\
&=\frac{1}{b^{2}\left(x_{c}\right)} \int_{x_{A}}^{x_{B}} d x_{c}^{\prime} c\left(x_{c}^{\prime}\right) h^{2}\left(x_{c}-x_{c}^{\prime}\right) b^{2}\left(x_{c}^{\prime}\right) .
\end{aligned}
$$

In our numerical studies in Sections III-B1 and III-C1, we have used $\nu_{m}=\left(1 / 2 \Delta_{c}\right)$, where $\Delta_{c}$ denote the sample interval of $\mathrm{g}\left(x_{c}, s_{a}, s_{b}\right)$.

6) Noise Properties of Chord Images: Using (4), one can write the variance of the reconstructed chord image as

$$
\begin{aligned}
\operatorname{Var} & \left\{\mathbf{f}_{c}\left(x_{c}, s_{a}, s_{b}\right)\right\} \\
\approx & \operatorname{Var}\left\{\hat{\mathbf{f}}\left(x_{c}, s_{a}, s_{b}\right)\right\}+\frac{\operatorname{Var}\left\{\mathbf{P}\left(u_{d 0}, v_{d 0}, s_{a}\right)\right\}}{4 \pi^{2} b^{2}\left(x_{c}\right)} \\
& \times\left[\frac{\sqrt{\left(l-x_{B}\right)\left(x_{A}+l\right)}}{l-x_{c}}+\frac{\sqrt{\left(l+x_{A}\right)\left(l+x_{B}\right)}}{l+x_{c}}\right]^{2} .
\end{aligned}
$$

Substituting (36) into (37) yields

$$
\begin{aligned}
\operatorname{Var} & \left\{\mathbf{f}_{c}\left(x_{c}, s_{a}, s_{b}\right)\right\} \\
\approx & \frac{1}{b^{2}\left(x_{c}\right)} \int_{x_{A}}^{x_{B}} d x_{c}^{\prime} c\left(x_{c}^{\prime}\right) h^{2}\left(x_{c}-x_{c}^{\prime}\right) b^{2}\left(x_{c}^{\prime}\right) \\
& +\frac{\operatorname{Var}\left\{\mathbf{P}\left(u_{d 0}, v_{d 0}, s_{a}\right)\right\}}{4 \pi^{2} b^{2}\left(x_{c}\right)} \\
& \times\left[\frac{\sqrt{\left(l-x_{B}\right)\left(x_{A}+l\right)}}{l-x_{c}}+\frac{\sqrt{\left(l+x_{A}\right)\left(l+x_{B}\right)}}{l+x_{c}}\right]^{2}
\end{aligned}
$$

which provides a formula for computing the chord-image variance.

\section{B. Numerical Studies of Noise Properties in Parallel-Beam Reconstruction}

Using the parallel-beam configuration in Fig. 1(b) and the parameters given in Table I, we calculated noiseless projections for the numerical phantom in Fig. 1(a). We have used an object-independent Gaussian noise model and an object-dependent Poisson-noise model in the numerical studies. For each noise model, we generated 10000 sets of noisy data by using noiseless data as the means. The standard deviation $\sigma_{0}$ of Gaussian noise used is $1.6 \%$ of the maximum value in the noiseless data,

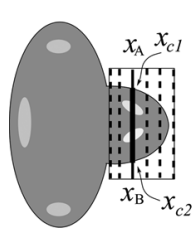

a

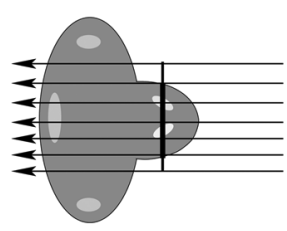

b

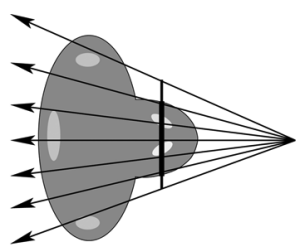

c
Fig. 1. (a) Phantom in numerical studies. (b) Parallel-beam configuration. (c) Fan-beam configuration. Solid line segment with endpoints $x_{A}$ and $x_{B}$ represents reconstruction segment. Thick segment with endpoints $x_{c 1}$ and $x_{c 2}$ indicates support segment. It should be noted that $\left[x_{A}, x_{B}\right] \supseteq\left[x_{c 1}, x_{c 2}\right]$. Rectangular ROI is decomposed into a set of (dashed) line segments.

whereas the standard deviation for the Poisson noise is the noiseless data scaled to yield a total photon count of $5 \times 10^{5}$ for each view. We investigated four reconstruction segments with different lengths $L_{A B}=\left|x_{B}-x_{A}\right|: 7.8,10.0,14.1$, and 20.0 $\mathrm{cm}$, all of which are located at $x=4.06 \mathrm{~cm}$. It can be observed in Fig. 1(b) that the length of the support segment, $5.5 \mathrm{~cm}$ in length, is shorter than the four reconstruction segments considered. Therefore, the image on this chord can be reconstructed exactly by use of data determined by these reconstruction segments. One can also conclude from Fig. 1(b) that the minimum data required by the first three reconstruction segments, which are shorter than the maximum dimension (about $15.6 \mathrm{~cm}$ ) of the object support, are truncated.

1) Noise Properties in Reconstruction from Truncated Parallel-Beam Data: From the 10000 sets of data containing Gaussian noise, we used (4)-(6) to reconstruct 10000 noisy $\mathbf{f}_{c}\left(x_{c}, s_{a}, s_{b}\right), \hat{\mathbf{f}}\left(x_{c}, s_{a}, s_{b}\right)$, and $\mathbf{g}\left(x_{c}, s_{a}, s_{b}\right)$, respectively, on the four reconstruction segments described previously. Based upon these noisy reconstructions, we subsequently computed their corresponding empirical variances, which are shown in the upper row of Fig. 2. We compare the empirical results to the analytical results obtained by use of (16), (36), and (38). The function $c\left(x_{c}\right)$ is determined by using $\sigma\left(u_{d}, s\right)=\sigma_{0}$ in (31), where $\sigma_{0}$ is $1.6 \%$ of the maximum value in noiseless data. The analytical results are displayed in the middle row of Fig. 2. Similarly, using (4)-(6), we reconstructed 10000 sets of noisy $\mathbf{g}\left(x_{c}, s_{a}, s_{b}\right), \hat{\mathbf{f}}\left(x_{c}, s_{a}, s_{b}\right)$, and $\mathbf{f}_{c}\left(x_{c}, s_{a}, s_{b}\right)$ on the four segments from 10000 sets of data containing Poisson noise. The computed empirical variances from these noisy images are displayed in the upper row of Fig. 3. Using the noiseless data as the Poisson-noise variance $\sigma^{2}\left(u_{d}, s\right)$ in (17), one can readily determine $c\left(x_{c}\right)$; and using the determined $c\left(x_{c}\right)$ in (16), (36), and (38), one can compute analytical image variances, which are displayed in the middle row of Fig. 3. The results show that the analytical and empirical results agree well with each other, suggesting that (38) provides an adequate estimation of the chord-image variance.

It can also be observed in Fig. 2(c) and Fig. 3(c) that the shorter the reconstruction segment, the higher the chord-image variances. This is only because the second term in (38) increases as $L_{A B}$ (i.e., $\left.\left(x_{A}-x_{c}\right)\left(x_{c}-x_{B}\right)\right)$ decreases. However, the difference of the chord-image variances in the central part of the support segment is quite small among these reconstruction segments. The implication of this result is that there may be a significant gain in terms of dose reduction by using a short 
TABLE I

Parameters for Circular Parallel-Beam, Circular Fan-Beam, and Helical Cone-BeAm Scanning Configurations Which ARe Used IN OUR Simulation Studies. SDD Is SOURCE-TO-Detector Distance AND SID DenOtes Source-To-Isocenter DisTance

\begin{tabular}{|c||c||c||c|}
\hline & Circular parallel-beam & Circular fan-beam & Helical cone-beam \\
& scan & scan & 300 \\
\hline Number of views per turn & 1024 & 512 & {$[-\pi, \pi]$} \\
Angular range & {$\left[-\frac{\pi}{2}, \frac{\pi}{2}\right]$} & {$[-\pi, \pi]$} & $128 \times 128$ \\
Detector bins & 256 & 256 & $3.1 \times 3.1$ \\
Detector bin size (mm) & 1.1 & 1.6 & 200 \\
SDD (mm) & - & 200 & 200 \\
SID (mm) & - & 200 & 90 \\
Helical pitch (mm) & - & - & \\
\hline
\end{tabular}
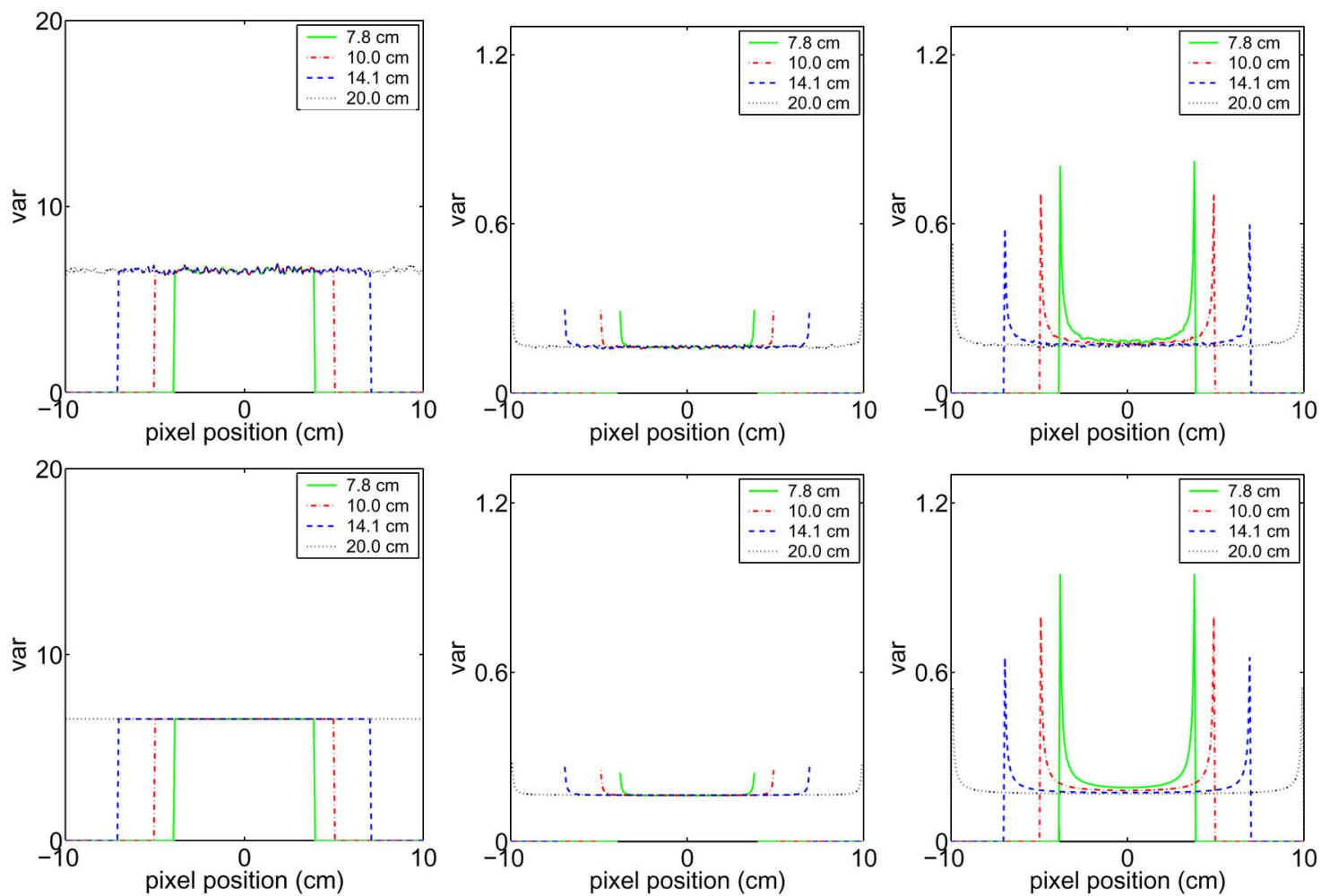

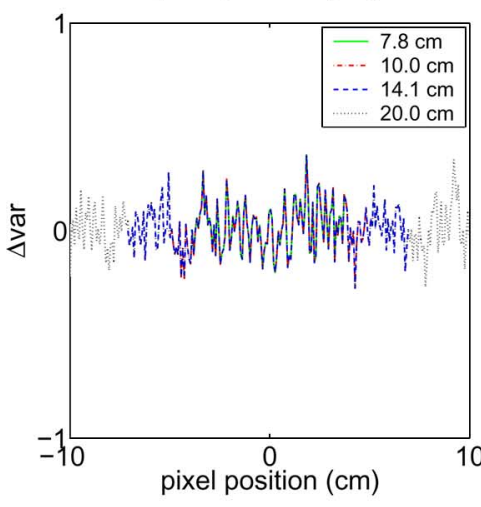

a

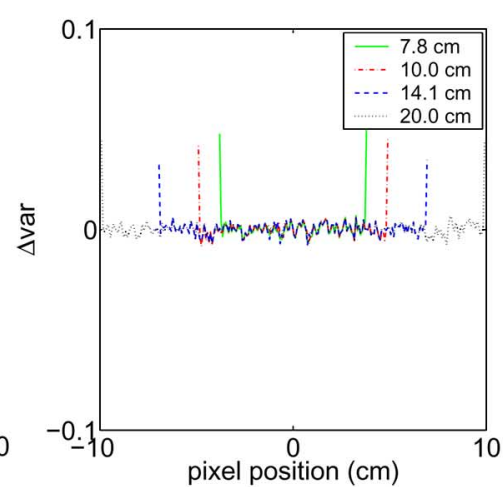

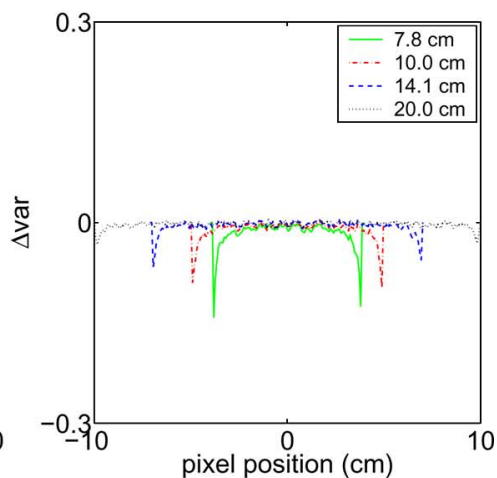

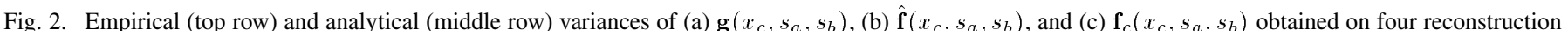

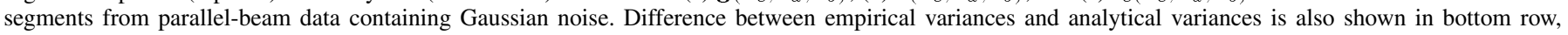

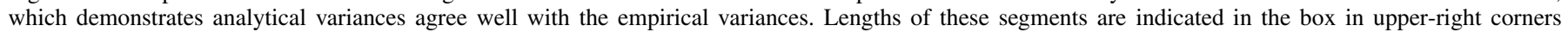
of plots.

reconstruction segment, because data required to reconstruct an image on this reconstruction segment is less than that required by using a longer reconstruction segment, thus resulting in a reduced illumination coverage to the object. For similar X-ray intensities, which are directly related to the data-noise level, the reconstruction using a short reconstruction segment appears to yield image variance within the support segment that is comparable to that obtained with a longer reconstruction segment. 

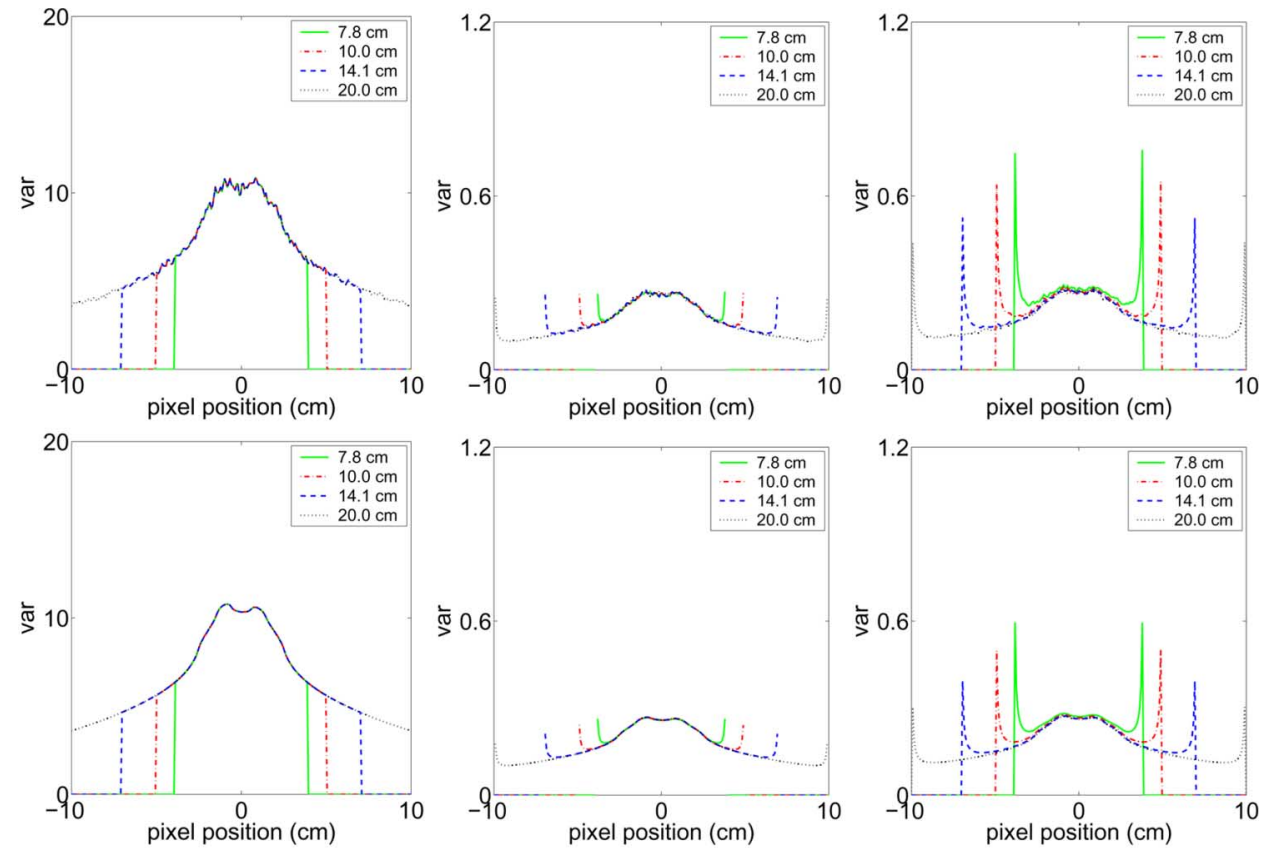

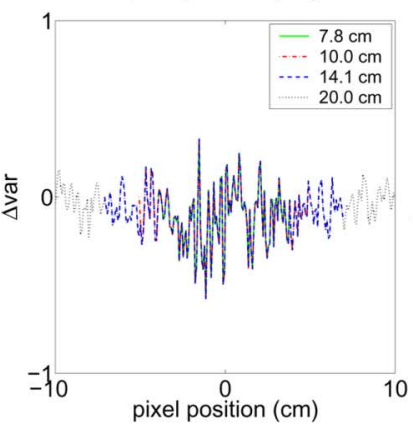

a

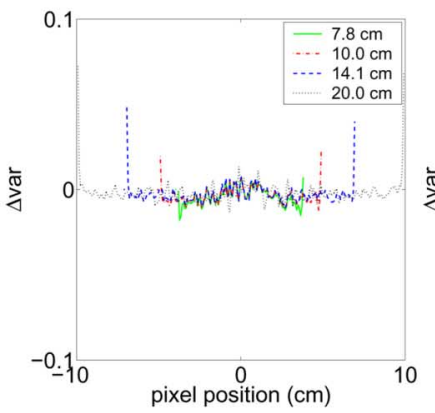

b

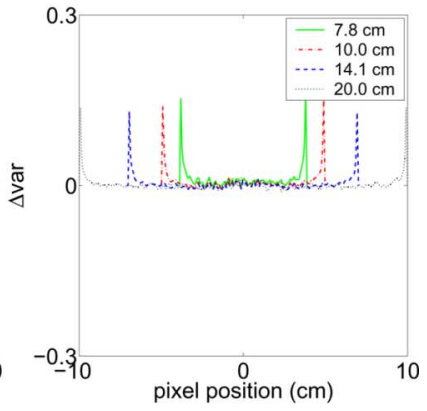

c

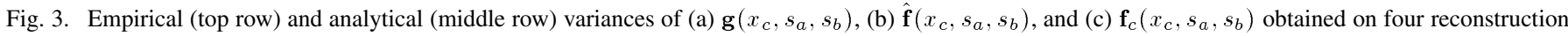
segments from parallel-beam data containing Poisson noise. Difference between empirical variances and analytical variances is also shown in bottom row, which demonstrates analytical variances agree well with empirical variances. Lengths of these segments are indicated in the box in upper-right corners of plots.

We have also performed numerical studies of the noise properties of the reconstructed ROI images by use of the BPF and MDFBP algorithms from truncated data. Using the numerical phantom in Fig. 1 and each of Gaussian- and Poisson-noise models described above, we generated 500 noisy, truncated data sets for image reconstruction on reconstruction segments of a length $L_{A B}=10.0 \mathrm{~cm}$, as shown in Fig. 1(a), which completely cover the ROI. We subsequently reconstructed 500 noisy images by using the BPF and MDFBP algorithms. We display in Fig. 4 noisy ROI images reconstructed using the BPF and MDFBP algorithms from data containing Gaussian noise (upper row) and Poisson noise (lower row).

Using the reconstructed 500 sets of Gaussian-noise images and 500 sets of Poisson-noise images, we computed empirical variances of the ROI images, which are shown in the upper row and lower row of Fig. 5, respectively. We display in the third column of Fig. 5 the variance profiles on the dashed lines indicated in the variance images. Results in Fig. 5 support the conclusion that both BPF and MDFBP algorithms yield images with comparable variance levels.

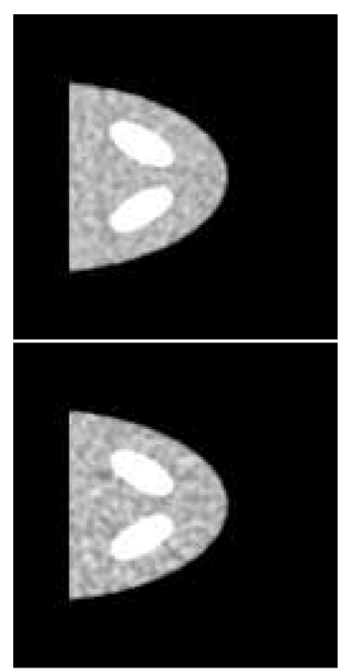

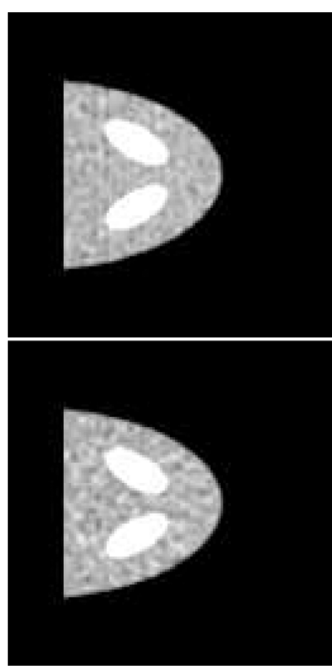

b
Fig. 4. ROI-images obtained by use of (a) BPF algorithm and (b) MDFBP algorithm from truncated parallel-beam data containing Gaussian noise (upper row) and Poisson noise (lower row). 

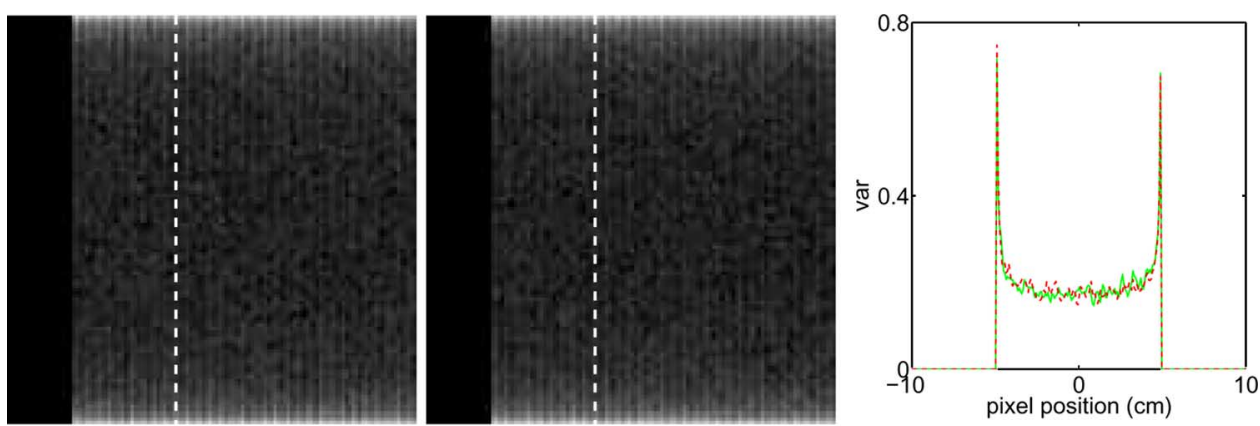

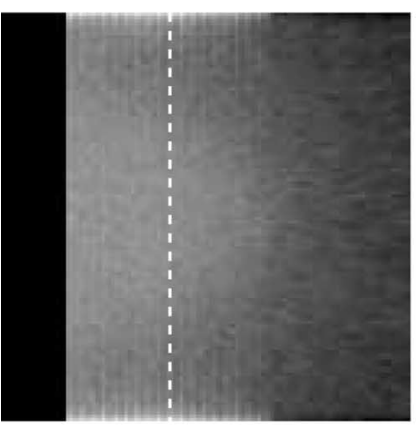

a

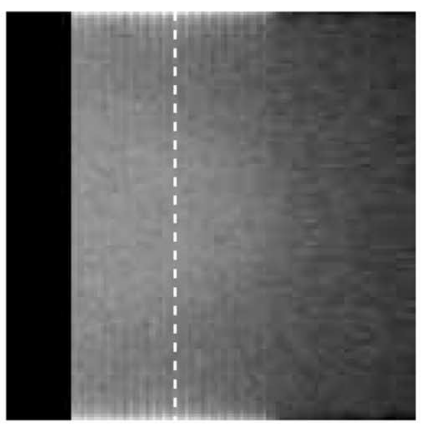

b

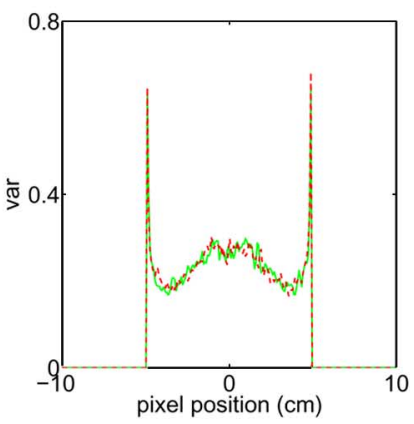

c

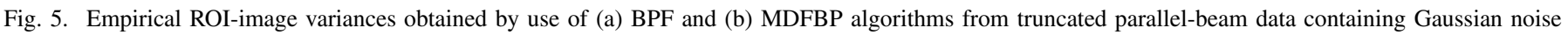

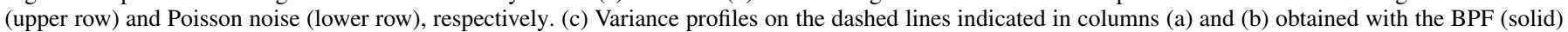

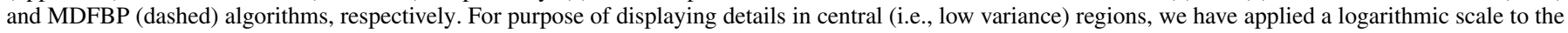
variance images. Display windows are $[-0.86,0.50]$ and $[-1.28,0.20]$ for Gaussian noise and Poisson noise, respectively.

2) Noise Properties in Reconstruction from Nontruncated Parallel-Beam Data: As discussed above, the FBP algorithm cannot reconstruct exactly images from truncated data. Therefore, we study the noise properties of the FBP algorithm from parallel-beam data without truncations. For the purpose of comparison, we have also included reconstruction results of the BPF and MDFBP algorithms from the same nontruncated data. Using the numerical phantom in Fig. 1 and each of the Gaussian- and Poisson-noise models, we generated 500 noisy data sets from which 500 noisy images were obtained by use of each of the BPF, MDFBP, and FBP algorithms. Using these noisy images, we computed empirical variance images, which are shown in the upper and lower rows of Fig. 6, respectively, for the Gaussianand Poisson-noise models. We also display in Fig. 7 the variance profiles on the dashed lines (i.e., on a chord) indicated on the variance images in Fig. 6. The profile results were obtained by use of the BPF (solid), MDFBP (dashed), and FBP (dotted) algorithms for the (a) Gaussian-noise model and (b) Poisson-noise model. It can be observed that image variances obtained with the three algorithms are similar and that the only difference comes at the extreme ends of the shown reconstruction segments. The $\mathrm{BPF}$ and MDFBP algorithms show a significant increase in the image variance at both ends of the profile. The reason for this is that the reconstruction segment was taken to be the width of the image array, and the prefactor for the finite Hilbert transform in (4) and (7) has a singularity at the ends of the reconstruction segment. In practical situations this prefactor is of little consequence because the reconstruction segment can be selected larger to avoid the singular behavior; furthermore, because the singularity goes as the $-1 / 2$ power, its effect is evident only very close to the endpoints of the reconstruction segment.

\section{Numerical Studies of Noise Properties in Fan-Beam Reconstruction}

Using the fan-beam configuration in Fig. 1(c) and the parameters listed in Table I, we calculated fan-beam noiseless data for the numerical phantom in Fig. 1(a). We have also used an object-independent Gaussian-noise model and an object-dependent Poisson-noise model in this numerical study. The standard deviation $\sigma_{0}$ of Gaussian noise used is $2.3 \%$ of the maximum value in noiseless fan-beam data, whereas the standard deviation for the Poisson noise is the noiseless data scaled to yield a total photon count of $5 \times 10^{5}$ for each view. For each noise model, 10000 sets of noisy data were generated by use of the corresponding noiseless data as the means. We investigated reconstruction segments of four different lengths $L_{A B}=$ $7.8,10.0,14.1$, and $20.0 \mathrm{~cm}$. All of the segments are located at $x=4.06 \mathrm{~cm}$. It can be observed in Fig. 1(c) that the length of the support segment is $5.5 \mathrm{~cm}$, which is shorter than the four reconstruction segments. Therefore, the image on this chord can be reconstructed exactly by use of data determined by these reconstruction segments. One can also conclude from Fig. 1(c) that data determined by the first three reconstruction segments, which are shorter than the maximum dimension (about $15.6 \mathrm{~cm}$ ) of the object support, are truncated.

1) Noise Properties in Reconstruction from Truncated FanBeam Data: From the 10000 sets of data containing Gaussian noise, we used (4)-(6) to reconstruct 10000 noisy $\mathbf{f}_{c}\left(x_{c}, s_{a}, s_{b}\right)$, $\hat{\mathbf{f}}\left(x_{c}, s_{a}, s_{b}\right)$, and $\mathbf{g}\left(x_{c}, s_{a}, s_{b}\right)$, respectively, on the four reconstruction segments described. Based upon these noisy reconstructions, we subsequently computed their corresponding empirical variances, which are shown in the upper row of Fig. 8. 

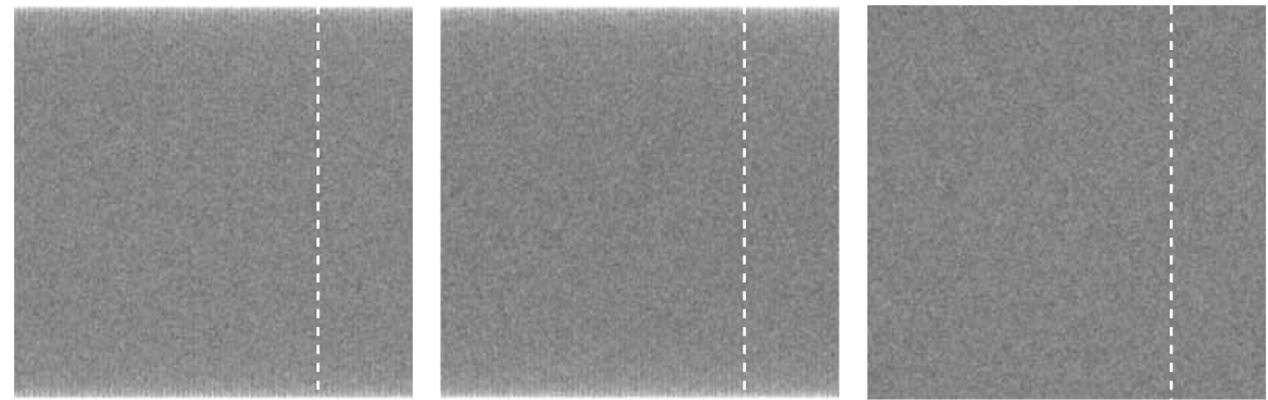

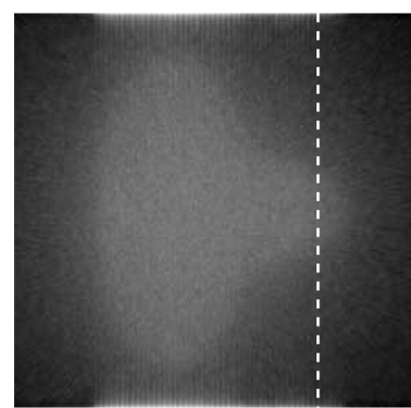

a

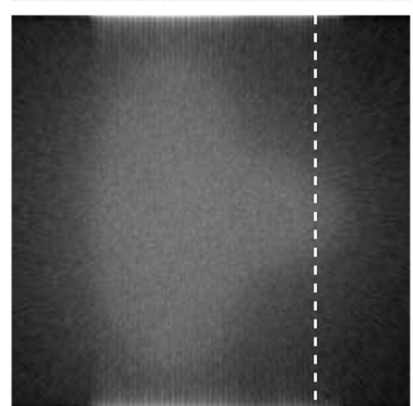

b

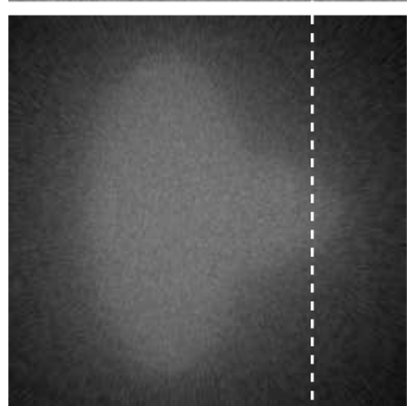

Fig. 6. Empirical variance images obtained by use of (a) BPF, (b) MDFBP, and (c) FBP algorithms from nontruncated parallel-beam data containing Gaussian noise (upper row) and Poisson noise (lower row). For the purpose of displaying details in central (i.e., low variance) regions, we have applied a logarithmic scale to variance images. Display windows are $[-1.3,0.30]$ and $[-1.3,0.40]$ for Gaussian noise and Poisson noise, respectively.

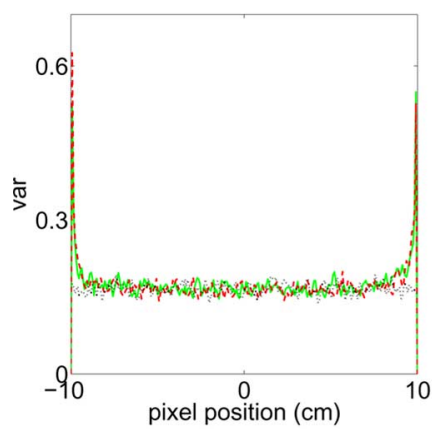

a

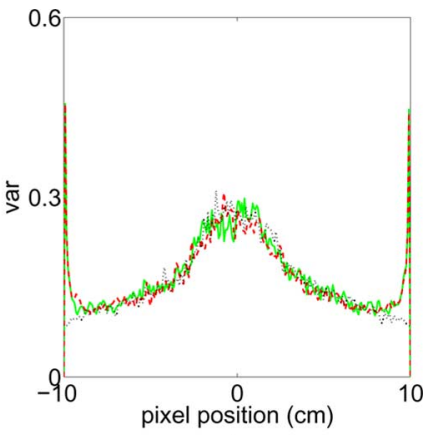

b
Fig. 7. Variance profiles on the dashed lines indicated in variances images shown in Fig. 6. They were obtained for the Gaussian (a) and Poisson (b) noise models by use of the BPF (solid), MDFBP (dashed), and FBP (dotted) algorithms, respectively.

As for the analytic variance, one can determine $c\left(x_{c}\right)$ by using $\sigma\left(u_{d}, s\right)=\sigma_{0}$ in (32), where $\sigma_{0}$ is $2.3 \%$ of the maximum value in noiseless fan-beam data. Using $c\left(x_{c}\right)$ in (23), (36), and (38), we computed analytically image variances, which are displayed in the middle row of Fig. 8. Similarly, using (4)-(6), we reconstructed 10000 sets of noisy $\mathbf{f}_{c}\left(x_{c}, s_{a}, s_{b}\right), \hat{\mathbf{f}}\left(x_{c}, s_{a}, s_{b}\right)$, and $\mathrm{g}\left(x_{c}, s_{a}, s_{b}\right)$ on the four segments from 10000 sets of fan-beam data containing Poisson noise. The computed empirical variances from these noisy images are displayed in the upper row of Fig. 9. Furthermore, using the noiseless fan-beam data as the Poisson-noise variance $\sigma^{2}\left(u_{d}, s\right)$ in (32), one can readily determine $c\left(x_{c}\right)$. Using the determined $c\left(x_{c}\right)$ in (23), (36), and (38), we computed analytically image variances, which are displayed in the middle row of Fig. 9. It can be observed that the analytic and empirical results agree well with each other, suggesting that (38) provides an adequate analytic estimation of the chord-image variance for the fan-beam case as well. It is interesting to note in Fig. 8(a) and Fig. 9(a) that the variances of $\mathbf{g}\left(x_{c}, s_{a}, s_{b}\right)$ are spatially varying on the chord. Based upon (32), one can readily conclude that this spatial variation is caused by the spatially variant factor $\left(A^{2}\left(u_{d}\right) /\left|\vec{r}-\vec{r}_{0}(s)\right|^{4}\right)$.

Again, from these results, observations similar to those for the parallel-beam case can be made for the fan-beam case. For example, as Fig. 8(c) and Fig. 9(c) show, the shorter the reconstruction segment, the higher the chord-image variances. This is only because the second term in (38) increases as $L_{A B}$ (i.e., $\left.\left(x_{A}-x_{c}\right)\left(x_{c}-x_{B}\right)\right)$ decreases. The implication of these results is that there may be a significant gain in terms of dose reduction by using a short reconstruction segment, because data required to reconstruct an image on this reconstruction segment is less than that required by using a longer reconstruction segment, which can result in reduction of illumination coverage of the object.

We have also performed numerical studies of the noise properties of the reconstructed ROI images by use of the BPF and MDFBP algorithms from truncated data. Using the numerical phantom in Fig. 1 and each of the Gaussian- and Poisson-noise models described previously, we generated 500 noisy truncated data sets for image reconstruction on reconstruction segments of a length $L_{A B}=10.0 \mathrm{~cm}$, as shown in Fig. 1(a), which cover the ROI completely. We subsequently reconstruct 500 noisy images by using each of the BPF and MDFBP algorithms. Using the reconstructed 500 sets of Gaussian-noise images and 500 sets of Poisson-noise images, we computed empirical variance images within the ROI, which are shown in the upper row and lower row of Fig. 10, respectively. Moreover, we display in the third column of Fig. 10 the variance profiles on the dashed lines 

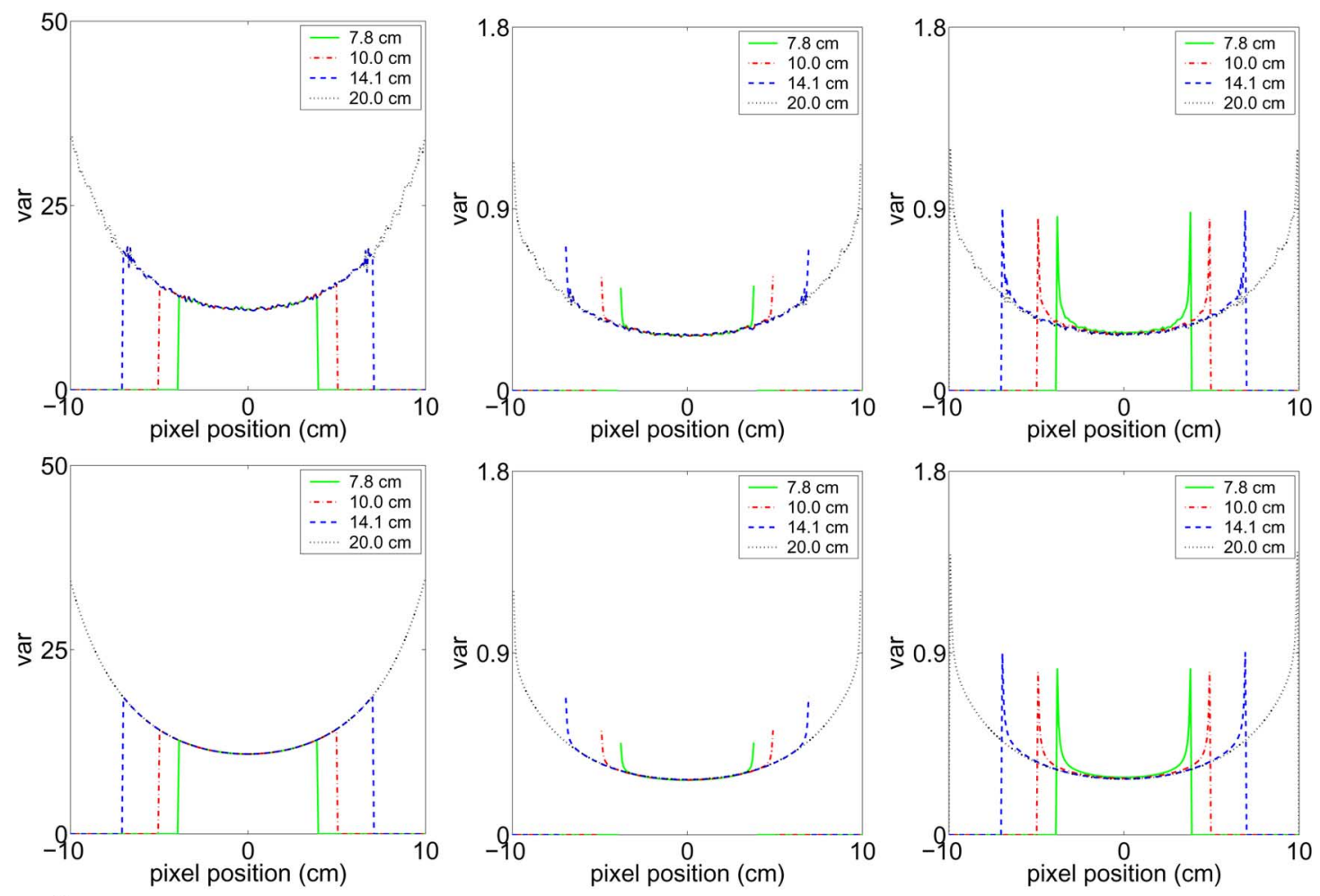

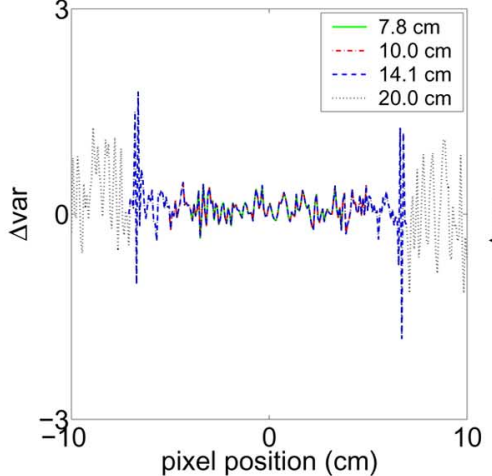

a

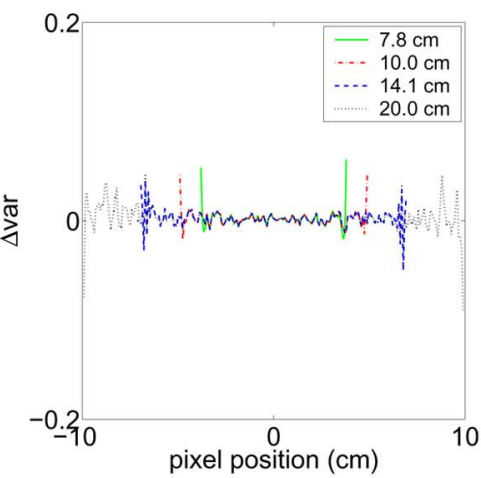

b

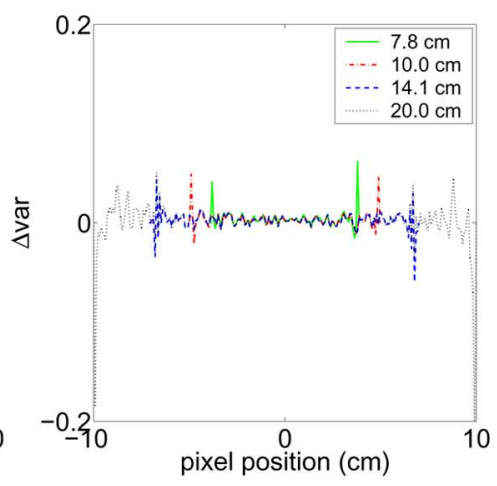

C

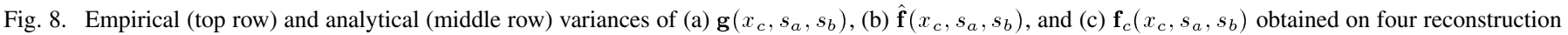

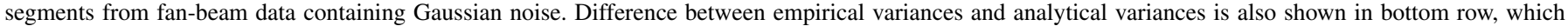
demonstrates the analytical variances agree well with the empirical variances. Lengths of these segments are indicated in the box in upper-right corners of plots.

indicated in the variance images. Results in Fig. 10 support the conclusion that both BPF and MDFBP algorithms yield images with comparable variance levels.

2) Noise Properties in Reconstruction From Nontruncated Fan-Beam Data: The FBP algorithm cannot reconstruct exactly images from truncated fan-beam data. Thus, we evaluate the noise properties of the FBP reconstruction from nontruncated fan-beam data. For the purpose of comparison, we have also included reconstruction results of the BPF and MDFBP algorithms from the same data sets. Using the numerical phantom and fan-beam configuration described above, we generated nontruncated fan-beam data at 512 views uniformly covering $2 \pi$. Using the noiseless data as the means, we generated 500 sets of data containing Gaussian noise and 500 sets of data containing Poisson noise. The standard deviation for the Gaussian noise is $2.3 \%$ of the maximum value of the noiseless data, whereas the standard deviation for the Poisson noise is the noiseless data scaled to yield a total photon count of $5 \times 10^{5}$ for each view. For a given chord specified by $s_{a}$ and $s_{b}$, one can reconstruct its image from data acquired over the right-side trajectory (i.e., $s \in\left[s_{a}, s_{b}\right]$ ), as shown in Fig. 11(a). Conversely, one can also reconstruct the chord image from data acquired with both right-side trajectory (i.e., $s \in\left[s_{a}, s_{b}\right]$ ) and left-side trajectory (i.e., $s \in\left[s_{b}, s_{a}\right]$ ), as shown in Fig. 11(b). In chord-based image reconstruction, we decompose image area into chords parallel to the vertical direction and the source scans from $s_{a}$ to $s_{b}$ and then from $s_{b}$ to $s_{a}$, as shown in Fig. 11 .

For each chord in the set covering the image area, we first reconstructed the images by use of the BPF, MDFBP, and FBP algorithms from data containing Gaussian noise acquired over the right-side trajectory specified by $s \in\left[s_{a}, s_{b}\right]$. Subsequently, we computed empirically chord-image variances from these noisy reconstructions. By assembling the chord-image variances, we obtain the variance images, which are shown in the upper row of 

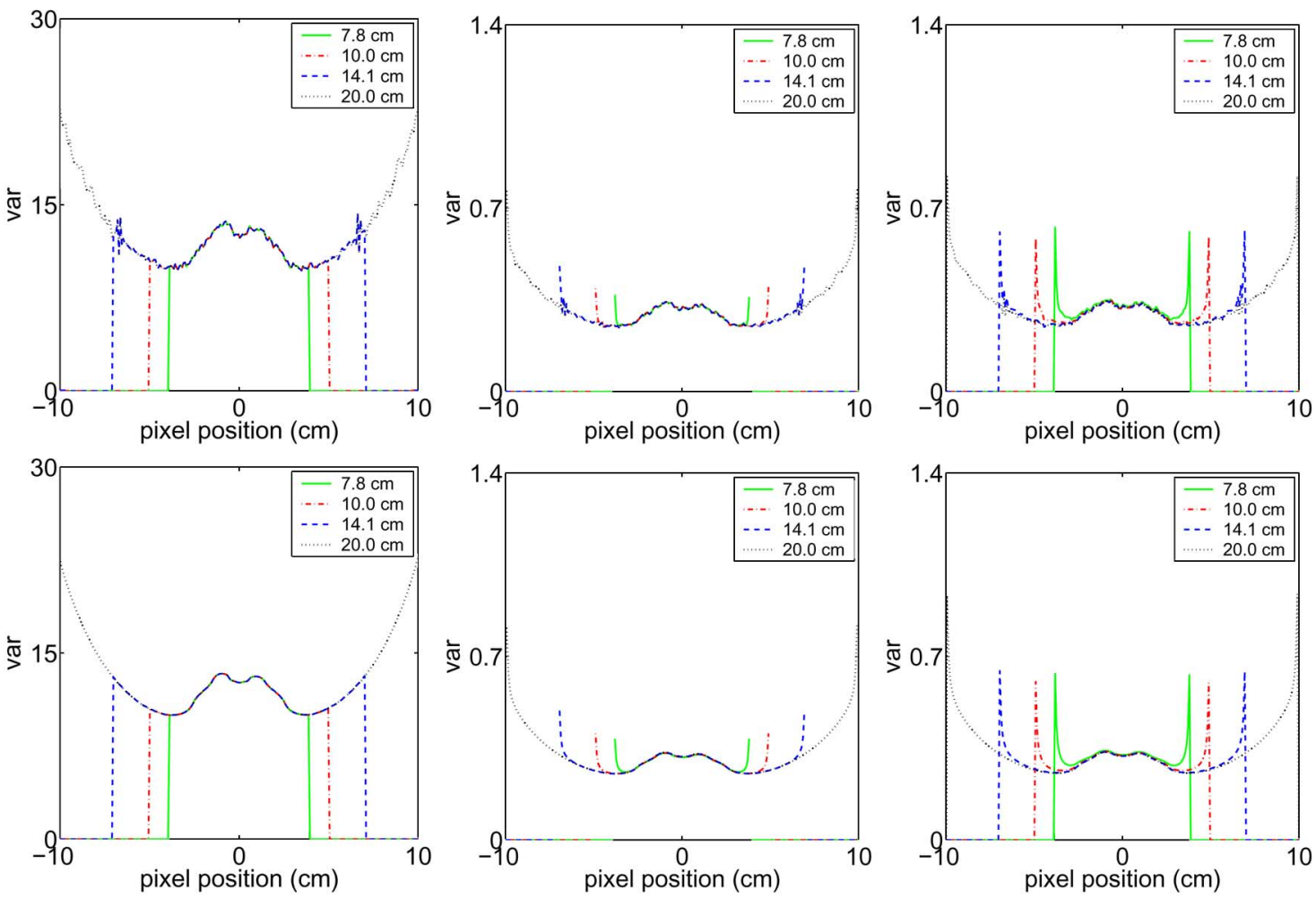

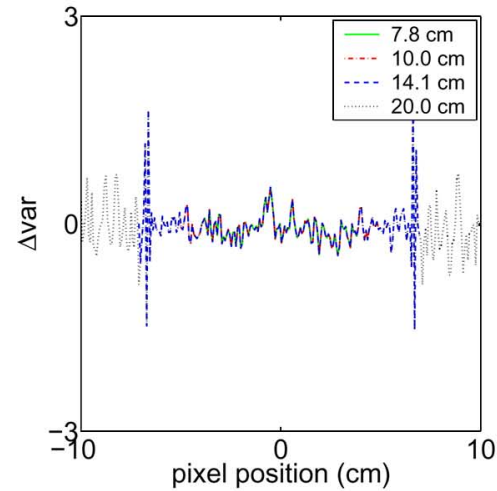

a

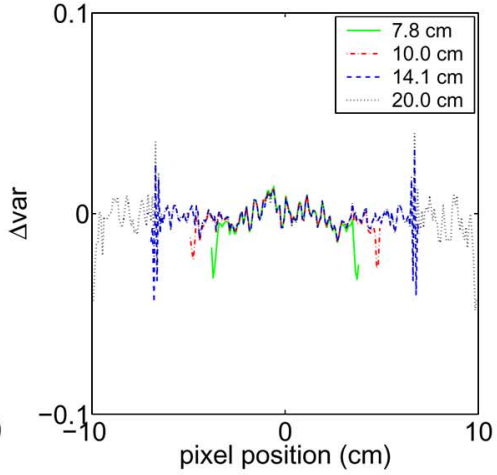

b

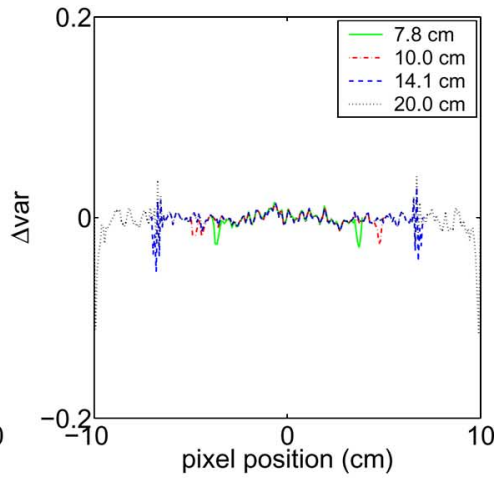

C

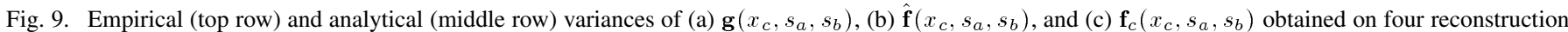
segments from fan-beam data containing Poisson noise. Difference between empirical variances and analytical variances is also shown in bottom row, which demonstrates analytical variances agree well with empirical variances. Lengths of these segments are indicated in the box in upper-right corners of plots.

Fig. 12, for the BPF, MDFBP and FBP algorithms, respectively. Similarly, from data containing Poisson noise, we obtained the image variances, which are displayed in lower row of Fig. 12.

We show in column one of Fig. 13 the image variances on a chord specified by $s_{a}=-\pi / 2$ and $s_{b}=\pi / 2$ obtained from data containing Gaussian noise (upper row) and Poisson noise (lower row), respectively. As already seen, the variance increases with the position along the chord near the source trajectory. There is little difference between the three algorithms. Furthermore, these variance images have similar properties: the chords on the right part have higher and more nonuniform image variance than those on the left part in the image area. In column 2 of Fig. 13, we show the profiles on the middle points across the vertical chords (i.e., on the middle horizontal lines in the variance images shown in Fig. 12). The results reveal that some difference of the MDFBP result from the BPF and FBP results in the peripheral region. This difference may be attributed to the data weighting prior to the backprojection step, which differs from that in the BPF and FBP algorithms. We should point out that this difference is only seen in the extreme periphery of the imaging area. For most practical situations these three algorithms perform virtually identically in terms of image variance.

We investigate further the general trend of the variance decreasing for chords on the left of the variance image. The first impression of this behavior is that this trend is obvious, because the scanning trajectory is on the right side, chords on the left of the variance image are reconstructed with a longer scanning trajectory. It appears that more data are used in reconstructing chords covering the left part of the variance image. This explanation is, however, incorrect. First, there is a slight upturn in the variance for the chords on the extreme left of the variance image, which runs counter to this trend. Second, 

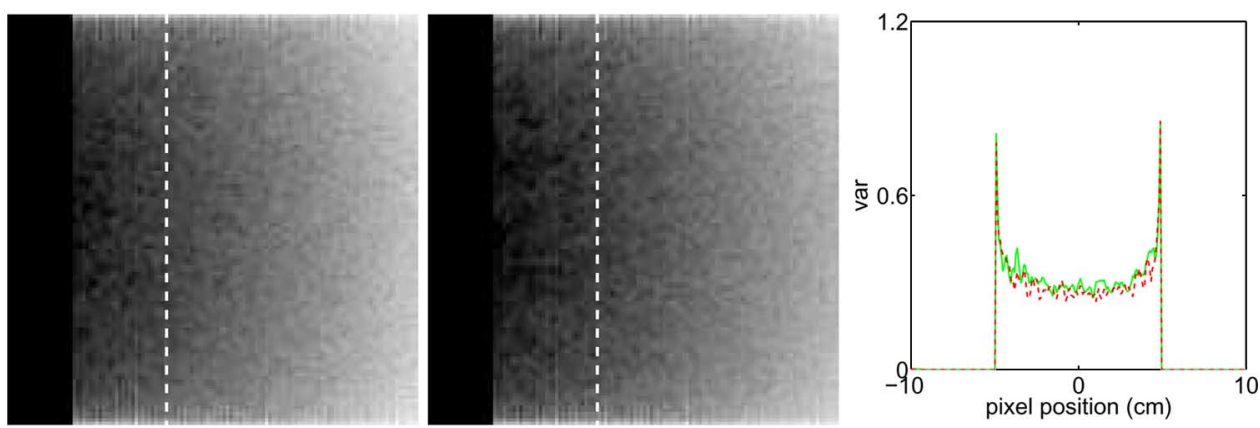

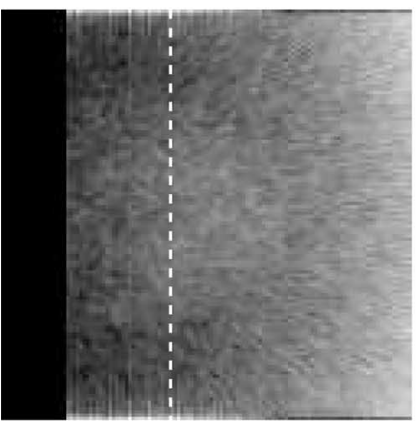

a

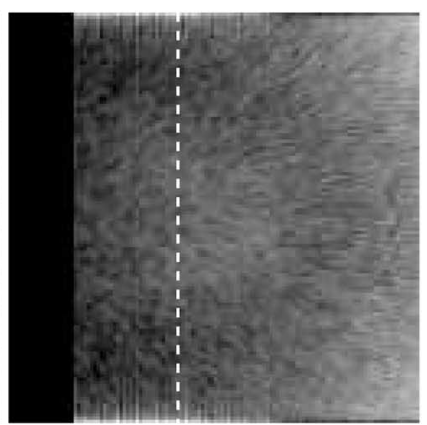

b

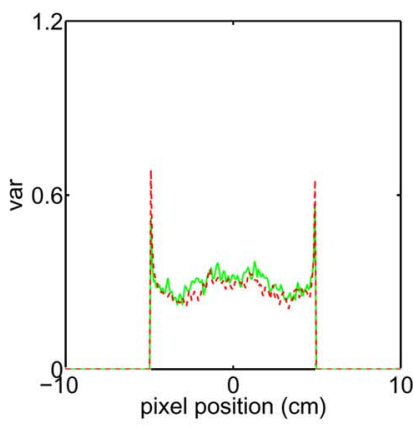

$\mathrm{c}$

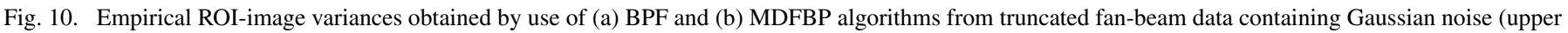

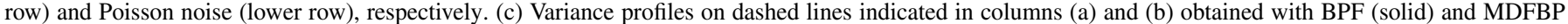

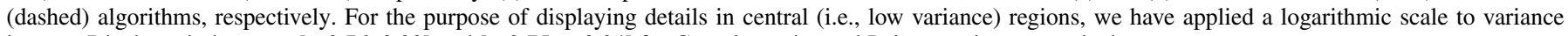
images. Display windows are $[-0.76,0.29]$ and $[-0.75,-0.04]$ for Gaussian noise and Poisson noise, respectively.

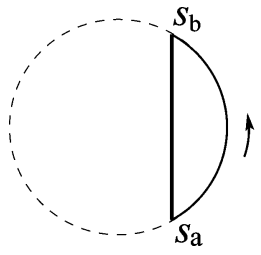

a

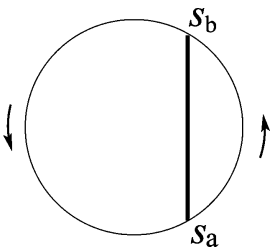

b
Fig. 11. (a) Right-side trajectory (solid) of chord (thick) specified by $s_{a}$ and $s_{b}$. (b) Right-side trajectory (solid) and left-side trajectory (solid) of chord (thick) specified by $s_{a}$ and $s_{b}$. Scanning configuration in (b) corresponds to a full fanbeam scan.

it can be demonstrated that the amount of data going into the chord reconstruction does not necessarily increase as the scanning trajectory increases. Based upon (32), one can conclude that the true cause of the variance behavior is spatially dependent weighting factor, $1 /\left|\vec{r}-\vec{r}_{0}(\lambda)\right|$, in the BPF, MDFBP, and FBP algorithms [19].

For a given chord specified by $s_{a}$ and $s_{b}$, when full scan data are available, one can reconstruct two chord images by use of data acquired with the right-side and left-side trajectories, as shown in Fig. 11 and then obtain a final chord image by averaging the two chord images. We show, in Fig. 14, the variance images of the full scan with accompanying profiles in Fig. 15.

\section{Numerical Studies of Noise Properties in Cone-Beam Reconstruction}

The BPF and MDFBP algorithms can yield exact image reconstruction on a chord specified by $s_{a}$ and $s_{b}$ as long as the support segment on the chord is illuminated by the X-ray beam at the projection views $s \in\left[s_{a}, s_{b}\right]$, because these algorithms require data only on the fan-beam projections of the support segment. From the perspective of the chord-based algorithms, the reconstruction of a chord image from cone-beam data is similar to that of a chord image from fan-beam data. In the fan-beam case, the orientation of the fan-beam planes at different views remain unchanged, whereas, in the cone-beam case with a non-planar trajectory, the orientation of the fan-beam-illumination planes generally varies from view to view. As discussed in Section III-A3, the noise properties of differentiation, backprojection, and filtration in the cone-beam case are similar to that in the fan-beam case. Therefore, we include below only the study results on the noise properties of the final chord-images reconstructed from cone-beam data.

1) Helical Cone-Beam Configuration: In our investigation of the noise properties of image reconstruction from cone-beam data, we consider the helical trajectory, which is the most widely used in clinical and industrial CT. For a helical scan, the source trajectory is described mathematically as $\vec{r}_{0}(s)=(R \cos s, R \sin s,(h / 2 \pi) s)$, where $R$ is the source to center-of-rotation distance, and $h$ indicates the helical pitch. For a chord specified by $s_{a}$ and $s_{b}$, if $(n-1) \pi \leq\left|s_{b}-s_{a}\right| \leq(n+1) \pi$, where $n$ is a positive odd integer, the chord is also referred to as an $n \pi$-line segment [20], [21], as shown in Fig. 16(a). In particular, when $n=1$ and thus $0 \leq\left|s_{b}-s_{a}\right| \leq 2 \pi$, the chord is referred to as a $\pi$-line segment [2], [11]. In this paper, we consider image reconstruction only on $\pi$-line segments for the reason that the imaging volume enclosed by the helix can be filled uniquely and completely by 

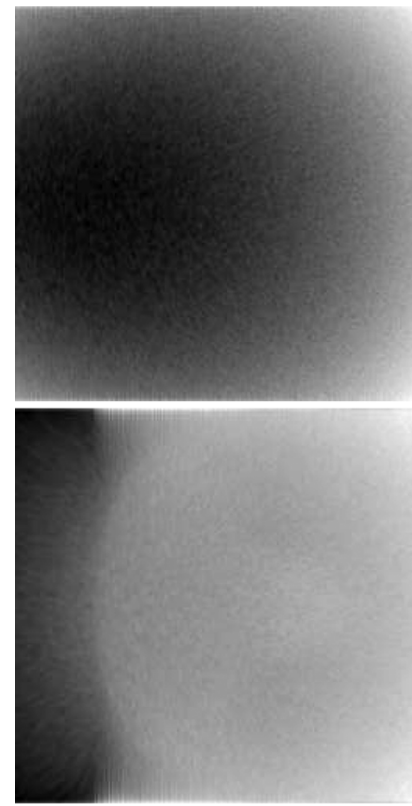

a
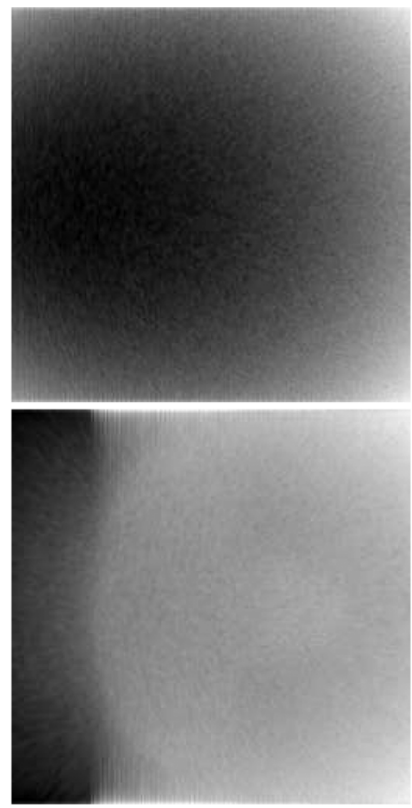

b
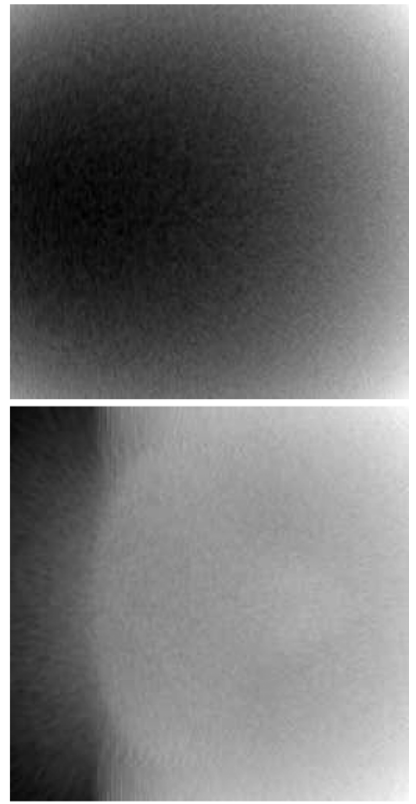

c

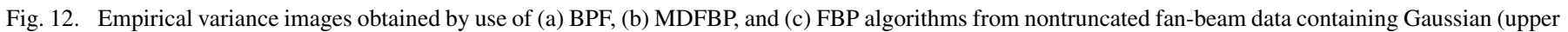

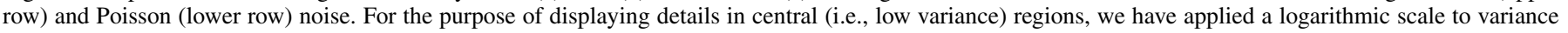
images. Display window is $[-1.0,0.65]$ and $[-2.02,0.29]$ for Gaussian noise and Poisson noise, respectively.
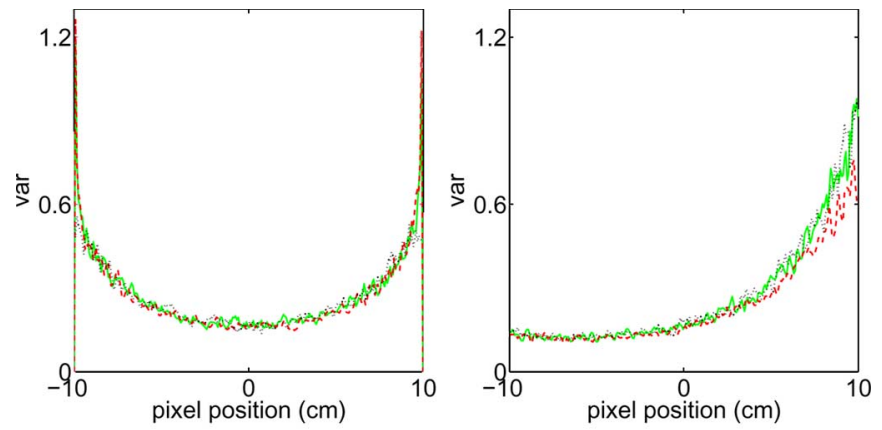

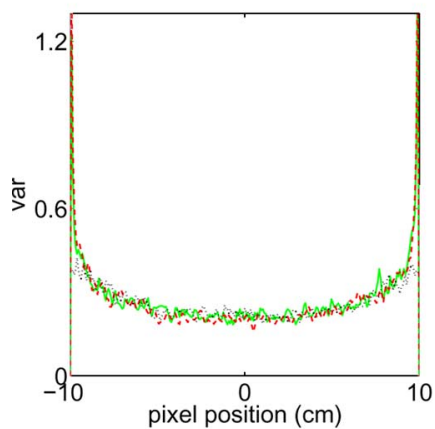

a

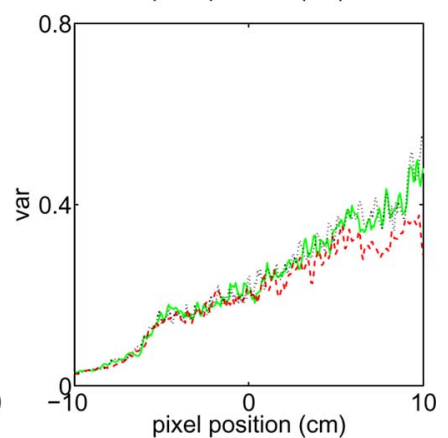

b
Fig. 13. Variance profiles along central (a) vertical and (b) horizontal lines in variance images shown in Fig. 12, obtained by BPF (solid), MDFBP (dashed), and FBP (dotted) algorithms from data containing Gaussian noise (upper row) and Poisson noise (lower row).

$\pi$-line segments [11], [12]. Thus, $\pi$-line segments can be used to form 3-D images in a helical cone-beam scan.

We computed the noiseless data from a Shepp-Logan phantom by use of the configuration parameters in Table I.
Using the noiseless data as the means, we subsequently generated 500 sets of data containing Gaussian noise and 500 sets of data containing Poisson noise, respectively. The standard deviation of Gaussian noise is chosen to be $0.7 \%$ of the maximum value of the noiseless data, whereas the standard deviation for the Poisson noise is the noiseless data scaled to yield a total count of $5 \times 10^{5}$ for each view.

2) Noise Properties in Reconstruction From Helical ConeBeam Data: A curved surface in the helix volume can be formed by a set of $\pi$-line segments for which we fix one end-point at $s_{a}$ and sweep the other endpoint over a range $s_{b} \in\left[s_{\min }, s_{\max }\right]$. We show in Fig. 16(a) a curved surface obtained by concatenating a set of $\pi$-line segments specified by $s_{a}=-\pi$ and $s_{b} \in[-0.5 \pi, 0.5 \pi]$. Using generated noisy helical cone-beam data, we reconstructed noisy images on the $\pi$-line surface by use of the BPF, MDFBP, and FBP algorithms. From these noisy images we subsequently computed empirical image variances on the $\pi$-line surface. In Fig. 17, we display the image variances obtained with BPF, MDFBP, and FBP algorithms from data containing Gaussian noise and Poisson noise.

We also display in Fig. 18(a) and (b) image variances on the $\pi$-line segment specified by $s_{a}=-\pi$ and $s_{b}=0$ in the surface, obtained from data containing Gaussian noise and Poisson noise, respectively. The image variances show similar characteristics to that of fan-beam image variances observed in Fig. 13. Namely, the variance image on the $\pi$-line surface in Fig. 17 has a structure that is similar to the right-side scan fan-beam results presented in Section III-C-1; the images on $\pi$-line segments reconstructed from smaller helix segments tend to have higher and more nonuniform variances. The similarity with the fan-beam case is not surprising because the geometrical arrangement of 

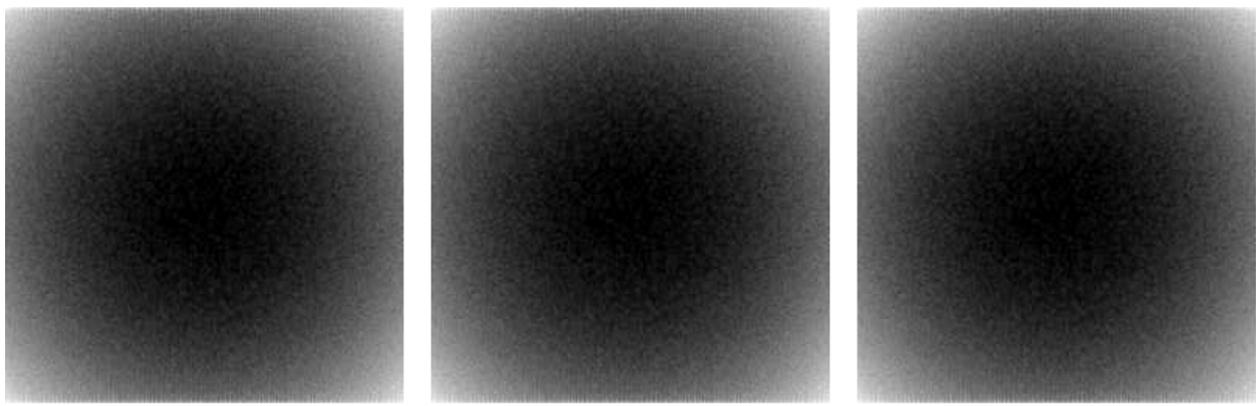

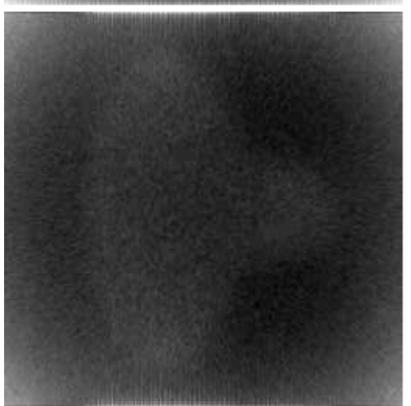

a

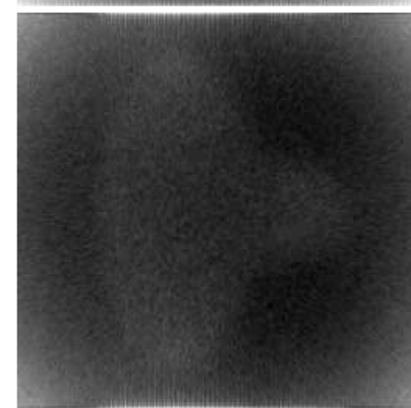

b

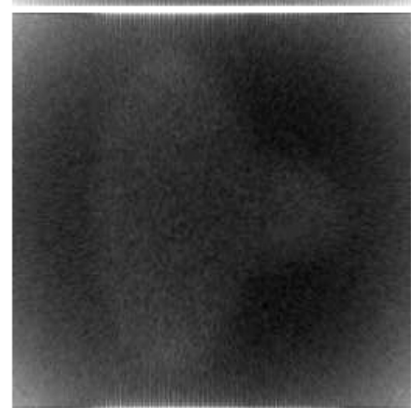

$\mathrm{c}$

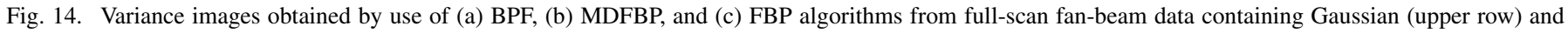

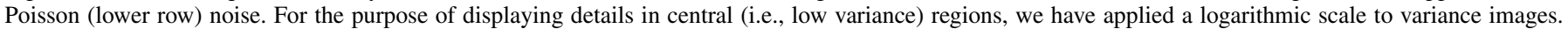
Display windows are $[-1.19,0.31]$ and $[-1.18,0.20]$ for Gaussian noise and Poisson noise, respectively.
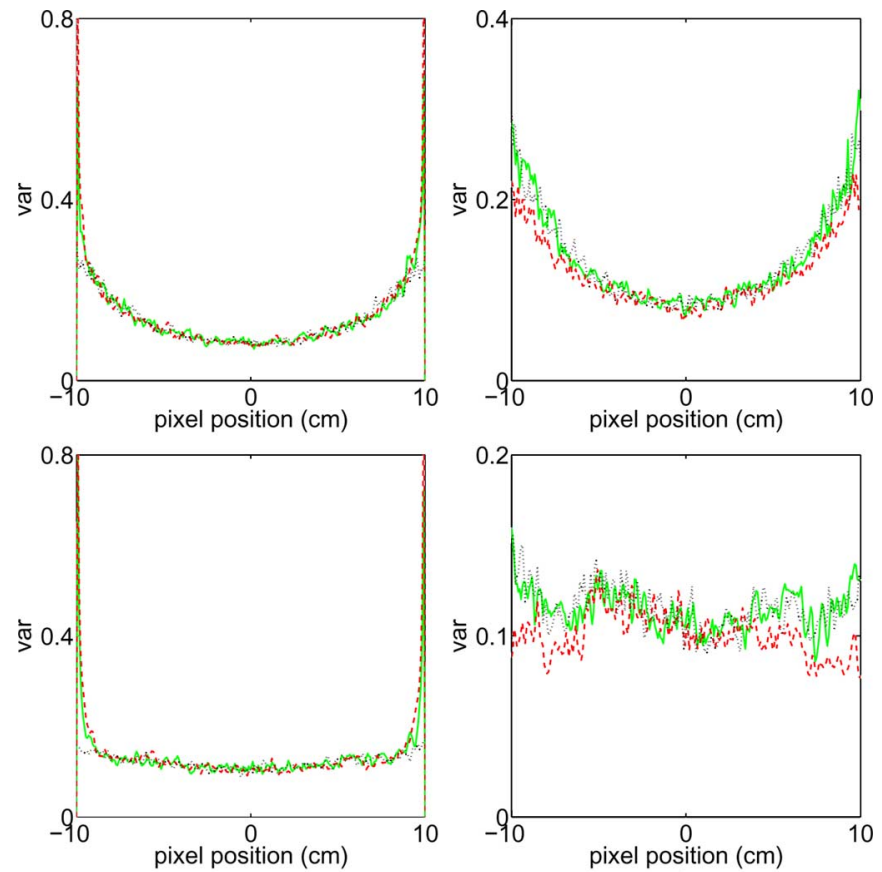

a

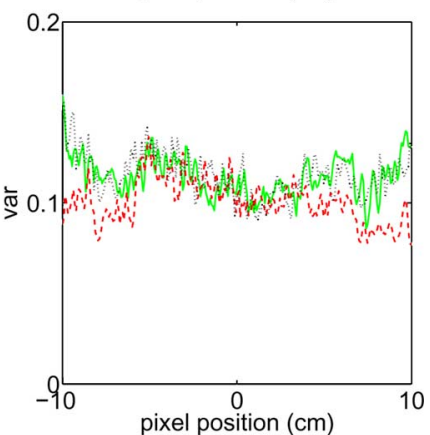

b

Fig. 15. Variance profiles along the central (a) vertical and (b) horizontal lines in variance images, which are shown in Fig. 14, obtained by BPF (solid), MDFBP (dashed), and FBP (dotted) algorithms from data containing Gaussian noise (upper row) and Poisson noise (lower row).

the $\pi$-line with respect to its scanning trajectory is very similar to the relationship between the chords and corresponding fan-beam scanning trajectory. The only difference is that there is an out-of-plane bent to the helix segment.

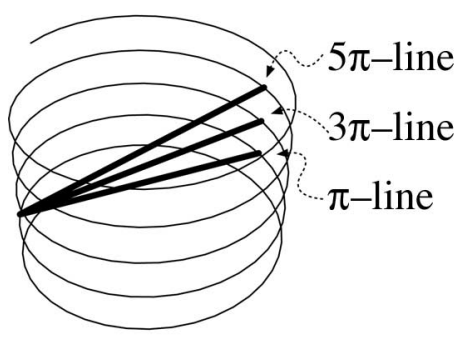

a

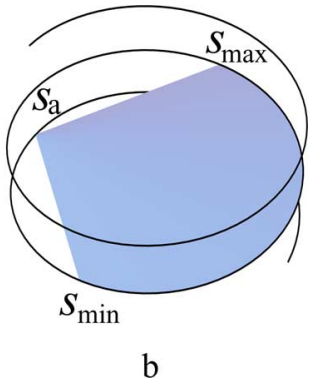

Fig. 16. (a) $\pi$-line, $3 \pi$-line, and $5 \pi$-line segments in a helical scan. (b) Surfaces generated in imaging volume by concatenating $\pi$-line segments specified by $s_{a}=-\pi$ and $s_{b} \in[-0.5 \pi, 0.5 \pi]$.

Regarding the nonuniform shape of the variance, one can attribute the high variance in the image periphery to the weighting factors multiplying the data derivatives before backprojection. As the algorithms are essentially the same for chord-image reconstruction in fan- and cone-beam cases, this conclusion should come as no surprise. In the 2-D fan-beam case, the variance nonuniformity and level was reduced by equally weighting reconstructions for both left and right side scans for each chord of the scanning circle. For the helical configuration, it is clear that in a typical scan there will be some overscan for nearly all the chords comprising the volume. But the overscan part of the trajectory does not form a closed loop so using the overscan data to reduce image variance is not as obvious as the case of the circular scan. Future work will focus on how to utilize the overscan data for non-closed trajectories for the purpose of reducing the impact of data noise on chord-base ROI-image reconstruction. 

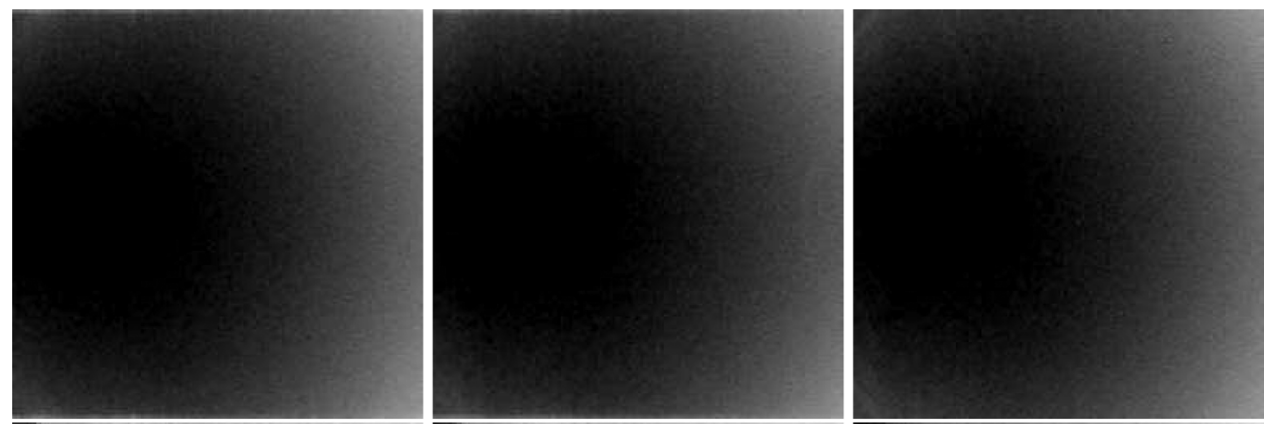

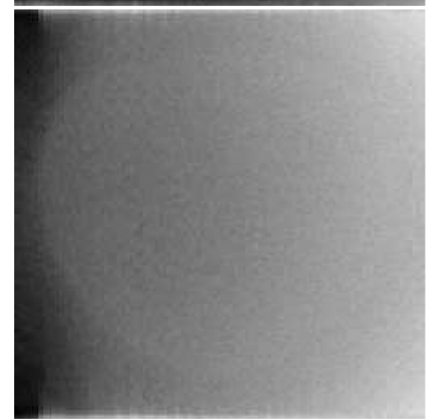

a

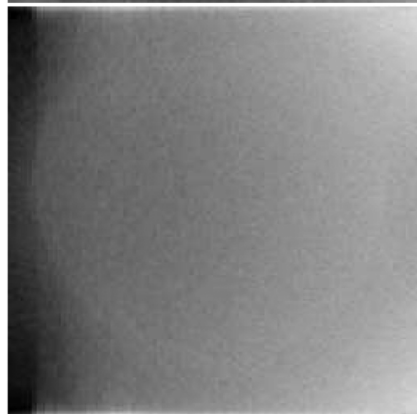

b

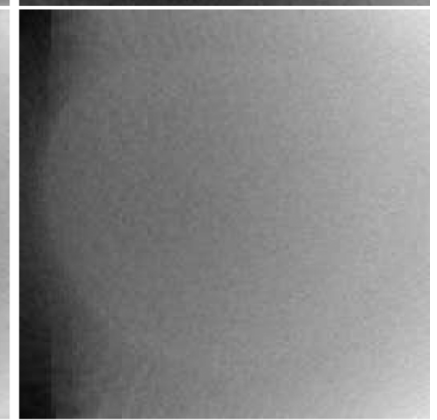

c

Fig. 17. Empirical variance images on $\pi$-line surface shown in Fig. 16(b) obtained by use of (a) BPF, (b) MDFBP, and (c) FBP algorithms from data containing Gaussian noise (upper row) and Poisson noise (lower row), respectively. For the purpose of displaying details in central (i.e., low variance) regions, we have applied a logarithmic scale to variance images. Display windows are $[-1.80,0.30]$ and $[-1.80,0.60]$ for Gaussian noise and Poisson noise, respectively.

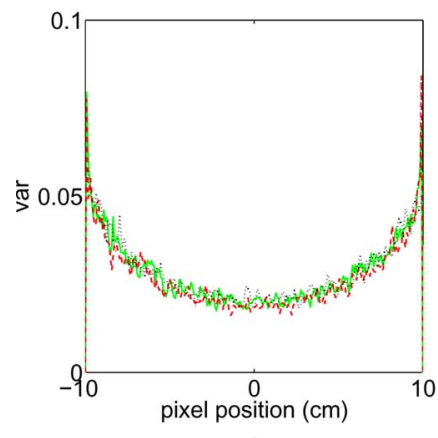

a

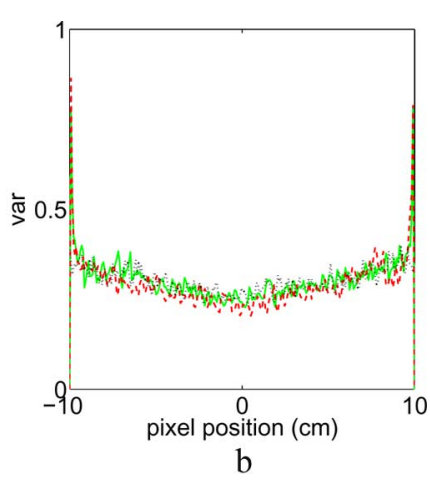

Fig. 18. Variances on central vertical line segment, specified by $s_{a}=-\pi$ and $s_{b}=0$, obtained with BPF (solid), MDFBP (dashed), and FBP (dotted) algorithms from data containing (a) Gaussian noise and (b) Poisson noise.

\section{DISCUSSION}

In this paper, we have performed analytic and numerical investigations of the noise properties of chord-based image reconstructions from parallel-, fan-, and cone-beam data. One of the main points of the investigation was to test whether or not the reduced illumination in designing a minimal data set for a particular ROI leads to a significant reduction in exposure. The idea was to compare the statistical properties of the ROI image reconstructed from noise realizations of the minimal data set with noise realizations of the full data set. Similar noise levels were used in both data sets, which are equivalent to modeling similar incident $\mathrm{X}$-ray beam intensities. Our study indicates that the resulting image variance was almost the same for images reconstructed from both data sets.
Thus, the minimal data set for ROI reconstruction leads to a significant overall dose reduction, because the body is exposed to lower amount of ionizing radiation in the reduced scan. For fan-beam and cone-beam imaging, we explored the noise properties of the extreme periphery of the imaging region by investigating large fan- and cone-angles. Image variance nonuniformity was found to be caused by spatially dependent weighting factors in the chord-based reconstruction algorithms. This work represents a study of the noise properties of chordbased reconstruction and of the impact of physical factors on ROI imaging in fan-beam and cone-beam CT. In seeking ways to reduce the impact of noise in volume imaging, we will investigate schemes to incorporate overscan data. The analysis presented in this work can directly be applied to chord-based image reconstruction for general trajectories. Finally, it is important to investigate the behavior of the ROI-reconstruction algorithms when other important factors are included in the data model such as X-ray polychromaticity and nonlinear partial volume averaging.

\section{REFERENCES}

[1] A. Katsevich, "Analysis of an exact inversion algorithm for spiral conebeam CT,” Phys. Med. Biol., vol. 47, pp. 2583-2597, 2002.

[2] Y. Zou and X. Pan, "Exact image reconstruction on PI-line from minimum data in helical cone-beam CT," Phys. Med. Biol., vol. 49, pp. 941-959, 2004.

[3] Y. Zou and X. Pan, "An extended data function and its backprojection onto PI-lines in helical conebeam CT," Phys. Med. Biol., vol. 49, pp. N383-N387, 2004.

[4] E. Y. Sidky, Y. Zou, and X. Pan, "Minimum data image reconstruction algorithms with shift-invariant filtering for helical, cone-beam CT," Phys. Med. Biol., vol. 50, pp. 1643-1657, 2005. 
[5] J. D. Pack, F. Noo, and R. Clackdoyle, "Cone-beam reconstruction using the backprojection of locally filtered projections," IEEE Trans. Med. Imag., vol. 24, no. 1, pp. 2317-2336, Jan. 2005.

[6] Y. Zou, X. Pan, and E. Y. Sidky, "Theory and algorithms for image reconstruction on chords and within regions of interest," J. Opt. Soc. Amer., vol. 22, pp. 2372-2384, 2005.

[7] S. Zhao, H. Yu, and G. Wang, "A unified framework for exact conebeam reconstruction formulas," Med. Phys., vol. 32, pp. 1712-1721, 2005.

[8] T. Zhuang, S. Leng, B. E. Nett, and G. Chen, "Fan-beam and cone-beam image reconstruction via filtering the backprojection image of differentiated projection data," Phys. Med. Biol., vol. 49, pp. 5489-5503, 2004.

[9] J. D. Pack and F. Noo, "Cone-beam reconstruction using 1D filtering along the projection of m-lines," Inv. Prob., vol. 21, pp. 1105-1120, 2005.

[10] Y. Zou and X. Pan, "Image reconstruction on PI-lines by use of filtered backprojection in helical cone-beam CT," Phys. Med. Biol., vol. 49, pp. 2717-2731, 2004.

[11] P. E. Danielsson, P. Edholm, and M. Seger, "Towards exact 3D-reconstruction for helical cone-beam scanning of long objects. A new detector arrangement and a new completeness condition," in Proc. 1997 Int. Meeting Fully Three-Dimensional Image Reconstruction Radiol. Nucl. Med., D. W. Townsend and P. E. Kinahan, Eds., Pittsburgh, PA, 1997, pp. 141-144.

[12] M. Defrise, F. Noo, and H. Kudo, "A solution to the long-object problem in helical cone-beam tomography," Phys. Med. Biol., vol. 45, pp. 623-643, 2000.
[13] X. Pan, Y. Zou, and D. Xia, "Peripheral and central ROI-image reconstruction from and data redundancy exploitation in truncated fan-beam data," Med. Phys., vol. 32, pp. 673-684, 2005.

[14] G. Chen, "An alternative derivation of Katsevich's cone-beam reconstruction formula," Med. Phys., vol. 30, pp. 3217-3226, 2003.

[15] F. Noo, M. Defrise, R. Clackdoyle, and H. Kudo, "Image reconstruction from fan-beam projections on less than a short scan," Phys. Med. Biol., vol. 47, pp. 2525-2546, 2002.

[16] Y. Ye and G. Wang, "Filtered backprojection formula for exact image reconstruction from cone-beam data along a general scanning curve," Med. Phys., vol. 32, pp. 654-665, 2005.

[17] X. Pan, D. Xia, Y. Zou, and L. Yu, "A unified analysis of FBP-based algorithms in helical cone-beam and circular cone- and fan-beam scans," Phys. Med. Biol., vol. 49, pp. 4349-4369, 2004.

[18] F. Noo, R. Clackdoyle, and J. Pack, "A two-step Hilbert transform method for 2D image reconstruction," Phys. Med. Biol., vol. 49, pp. 3903-3923, 2004.

[19] X. Pan and L. Yu, "Image reconstruction with shift-variant filtration and its implication for noise and resolution properties in fan-beam computed tomography," Med. Phys., vol. 30, pp. 590-600, 2003.

[20] R. Proksa, T. Köhler, and J. Timmer, "The n-PI-method for helical conebeam CT," IEEE Trans. Med. Imag., vol. 19, no. 7, pp. 848-863, Jul. 2000.

[21] C. Bontus, T. Köhler, and R. Proksa, "A quasiexact reconstruction algorithm for helical CT using a 3-Pi acquisition," Med. Phys., vol. 30, pp. 2493-2502, 2003. 